\title{
Energetic Oxygen and Sulfur Ions in the Jovian Magnetosphere and Their Contribution to the Auroral Excitation
}

\author{
N. Gehrels ${ }^{1}$ and E. C. Stone
}

\author{
California Institute of Technology, Pasadena, California 91125
}

\begin{abstract}
Observations of 1 to $20 \mathrm{MeV} / \mathrm{nuc}$ oxygen, sodium, and sulfur ions in the Jovian magnetosphere are reported. Measurements made by the cosmic ray subsystem on Voyager 1 and 2 are used to calculate abundances and energy spectra in the region from 5 to 20 Jovian radii $\left(R_{J}\right)$. The phase space density of the oxygen ions calculated from the spectra has a positive radial gradient between 6 and $17 R_{J}$, indicating an inward diffusive flow. The upper limit for the diffusion coeflicient $D$ at $9 R_{J}$ is $\sim 10^{-5} \mathrm{~s}^{-1}$. This limit, combined with the analysis of Voyager plasma observations by Siscoe et al. (1981), implies an upper limit to the production rate of oxygen and sulfur ions from Io of $\sim 10^{28}$ ions/s. If $D\left(9 R_{J}\right)$ is $\sim 4 \times 10^{-6} \mathrm{~s}^{-1}$, then $\sim 2 \times 10^{24}$ oxygen and sulfur ions with $>70 \mathrm{MeV}$ /nuc-G are lost per second as they diffuse inward from 12 to $8 R_{J}$. Assuming these ions are scattered into the loss cone, they deliver $\sim 4 \times 10^{12} \mathrm{~W}$ to the Jovian atmosphere. Extrapolations to lower magnetic moments suggest that the $10^{13}-10^{14} \mathrm{~W}$ required to produce the observed ultraviolet auroral emissions could result from the precipitation of $\sim 10^{26}$ oxygen and sulfur ions/s with magnetic moments $\gtrsim 10$ to $30 \mathrm{MeV} /$ nuc-G ( 235 to $100 \mathrm{keV} / \mathrm{nuc}$ at $10 R_{J}$ ). The ions with $\gtrsim 70$ $\mathrm{MeV} /$ nuc-G deposit their energy between $\sim 67^{\circ}$ and $\sim 72^{\circ}$ magnetic latitude at an average depth of $\sim 10^{19}$ $\mathrm{cm}^{-2}$ of $\mathrm{H}_{2}(\sim 500-\mathrm{km}$ altitude), which is above the homopause. If the extrapolated spectrum extends down to $\sim 10 \mathrm{MeV} / \mathrm{nuc}-\mathrm{G}$, then 10 times more energy $\left(\sim 10^{13} \mathrm{~W}\right)$ is carried inward across $10 R_{J}$ by the energetic oxygen and sulfur ions than flows outward with the plasma, indicating the presence of an energy source in the middle or outer magnetosphere.
\end{abstract}

\section{INTRODUCTION}

The auroral activity on Jupiter, discovered with the ultraviolet spectrometer on Voyager 1 [Broadfoot et al., 1979], is one of the most energetic phenomena associated with the Jovian magnetosphere, requiring a continuous power input of $10^{13}$ to $10^{14} \mathrm{~W}$ into the auroral zone [Broadfoot et al., 1981; Yung et al., 1982]. There has been wide interest [Thorne and Tsurutani, 1979; Goertz, 1980; Eviatar and Siscoe, 1980; Shemansky, 1980a, b; Thorne, 1981a, b, 1982; Sullivan and Siscoe, 1981] in determining the source of this power. A possible energy source that has been considered is the acceleration of Iogenic oxygen and sulfur ions to corotation speeds by Jupiter's magnetic field. This energy would be transported to the Jovian atmosphere by the local heating of torus electrons, followed by pitch angle scattering of the electrons into the loss cone [Thorne and Tsurutani, 1979]. Newly ionized oxygen and sulfur ions acquire 260 and $520 \mathrm{eV}$, respectively, in the corotating frame, so that $\gtrsim 2 \times 10^{29}$ ions/s would have to be created (the mass loading rate) in order to produce the auroral power.

Other observations of the Io plasma torus, however, indicate a lower mass injection rate. For example, in situ plasma observations [Bagenal and Sullivan, 1981] indicate that the torus contains $N \sim 5 \times 10^{34}$ ions, while the ionization states of the ions indicate that their diffusive lifetime $\tau_{\text {dirf }}$ is in the range from 9 to 600 days, with a nominal value of 100 days [Shemansky, $1980 b$; Shemansky and Sandel, 1982]. Thus the Io source rate $S_{i}$, which is $N / \tau_{\text {diff }}$ is in the range $10^{27} \leqslant S_{i} \leqslant 6 \times 10^{28}$ ions/s, with a nominal value of $6 \times 10^{27}$ ions/s. Thus the mass loading rate is apparently too low to provide the required power for the aurora.

Another possible energy source is the precipitation of inwardly diffusing energetic ions [Goertz, 1980; Thorne, 1981a,b,

\footnotetext{
${ }^{1}$ Currently at NASA Goddard Space Flight Center, Greenbelt, Maryland 20771.

Copyright 1983 by the American Geophysical Union.

Paper number 3 A0564.

0148-0227/83/003A-0564\$05.00
}

1982] instead of torus electrons. Thorne [1981a] has further suggested that auroral secondary electrons may be responsible for heating the torus. In this case, the energy is supplied by the process that accelerates the energetic particles in the outer magnetosphere and by the adiabatic acceleration of the ions as they diffuse inward. If, for instance, the acceleration in the outer magnetosphere occurs via a magnetic pumping process [Goertz, 1978; Borovsky et al., 1981], then the ultimate energy source is Jupiter's rotational energy. One of the goals of the present study is to determine the power delivered to the Jovian atmosphere by the precipitation of inwardly diffusing energetic oxygen and sulfur ions.

This paper is based on observations of oxygen, sodium, and sulfur ions with $\mathrm{MeV} /$ nuc energies made inside $\sim 20$ Jovian radii $\left(R_{J}\right)$ by the cosmic ray subsystem (CRS) on board Voyager 1 and 2. In previous reports [Vogt et al., 1979a,b; Gehrels et al., 1981, hereafter referred to as paper 1] it was found that the dominant energetic species with nuclear charge greater than 2 in the inner magnetosphere are oxygen and sulfur, with sodium also present. These abundances suggest that Io is the source of the ions. The radial gradient of the oxygen phase space density between 5 and $17 R_{J}$ was found to be positive indicating an inward diffusive flow in this region. It was postulated from these observations that plasma ions diffuse outward from the Io torus, are nonadiabatically accelerated in the outer magnetosphere, and then diffuse inward and outward from the acceleration region as energetic ions. In the present analysis, the oxygen phase space density is calculated with improved radial resolution and is used to set limits on the diffusion coefficient between 5 and $20 R_{J}$ and thereby on the mass loading rate of ions from Io. The inward diffusive flow rate of both oxygen and sulfur ions is determined, and the observed loss rate of the ions is used to calculate the power they deliver to the Jovian atmosphere.

\section{THE INSTRUMENT}

The data used in this analysis were obtained with CRS low-energy telescopes (LET's) [Stone et al., 1977; Stilwell et al., 


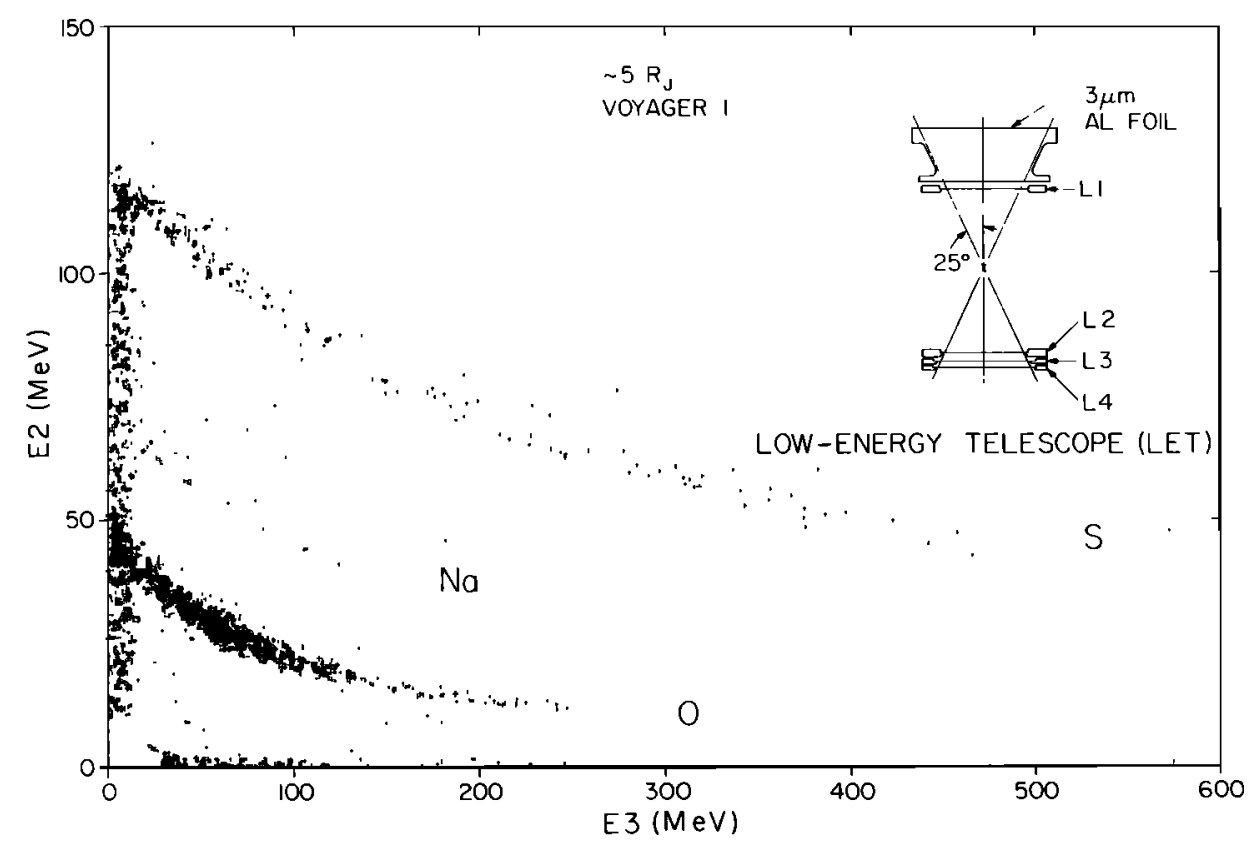

Fig. 1. The energy loss in detectors $L 2$ and $L 3$ of individual ions detected by LET D from day 64,0936 to 1405 UT (the 4.9 and 5.3 $R_{J}$ regions in Table 1). The oxygen, sodium, and sulfur tracks are indicated. Events in the bottom left-hand corner have not been plotted. The inset is a schematic diagram of a LET.

1979]. The instrument command state and telescope orientations in this region are described in paper 1 , with the one difference that only data from LET B were used in that study, while this analysis includes data from both LET B and LET D. A schematic diagram of a LET is shown in the inset of Figure 1. The LET's provide multiparameter analysis of individual nuclei with $\mathrm{MeV} /$ nuc energies and nuclear charge $Z$ greater than 2, with an rms charge resolution in the inner Jovian magnetosphere of $\sigma_{z} \approx 0.2$ charge units. Figure 1 shows the energy loss in detectors L2 and L3 of individual ions detected near 5 $R_{J}$. The large abundance of oxygen and sulfur and the presence of sodium in this region can be clearly seen.

In addition to the analysis of individual ions, the LET's also provide measurements of the counting rate of $Z>2$ ions, called the $Z>2$ rate, which is primarily the rate of particles losing more than $9.6 \mathrm{MeV}$ in $\mathrm{Ll}$ (paper 1). In regions where oxygen and sulfur are the dominant energetic species, this rate is approximately an integral measurement of oxygen and sulfur ions with energies greater than $\sim 1.1$ and $\sim 0.9 \mathrm{MeV} /$ nuc, respectively (energy loss in the 3- $\mathrm{mm}$ aluminum window of the LET is included in these thresholds). Therefore if the sulfur to oxygen ratio can be determined, the $Z>2$ rate can be used as an additional point in the integral energy spectra of either species.

\section{ENERGy SPECTRA AND AbUNDANCES}

Energy spectra were determined from the analyzed events in the 12 regions listed in Table 1 . The regions were chosen to

TABLE 1. Region Definitions and Magnetic Field Values Used in Spectral Analysis

\begin{tabular}{|c|c|c|c|c|}
\hline $\begin{array}{l}\text { Region, } \\
R_{J}\end{array}$ & Voyager & $\begin{array}{l}\text { Spacecraft Event } \\
\text { Time, } f \text { UT }\end{array}$ & $\begin{array}{c}\text { Magnetic Equatorial } \\
\text { Plane Crossing Time, } \\
\text { UT }\end{array}$ & $\begin{array}{l}\text { B Field at } \\
\text { Crossing, } \ddagger \\
\text { G }\end{array}$ \\
\hline 4.9 & 1 & 64,1106 to day 64,1235 & day 64,1200 & $3.26 \times 10^{-2}$ \\
\hline 5.3 & 1 & $\begin{array}{l}\text { day } 64,0936 \text { to day } 64,1106 \\
\text { day } 64,1235 \text { to day } 64,1405\end{array}$ & $\begin{array}{l}\text { day } 64,1034 \\
\text { day } 64,1341\end{array}$ & $2.79 \times 10^{-2}$ \\
\hline 5.9 & 1 & $\begin{array}{l}\text { day } 64,0842 \text { to day } 64,0936 \\
\text { day } 64,1405 \text { to day } 64,1443\end{array}$ & $\begin{array}{l}\text { day } 64,0905 \\
\text { day } 64,1428\end{array}$ & $2.08 \times 10^{-2}$ \\
\hline 7.9 & 1 & day 64,0546 to day 64,0659 & day 64,0608 & $7.18 \times 10^{-3}$ \\
\hline 10.1 & 2 & day 190,2214 to day 191,0016 & day 190,2300 & $3.34 \times 10^{-3}$ \\
\hline 12.2 & 2 & day 190,1235 to day 190,1440 & day 190,1338 & $1.74 \times 10^{-3}$ \\
\hline 13.0 & 1 & day 63,2328 to day 64,0110 & day 64,0008 & $1.32 \times 10^{-3}$ \\
\hline 13.7 & 2 & day 190,0907 to day 190,1109 & day 190,1008 & $1.20 \times 10^{-3}$ \\
\hline 16.8 & 1 & day 63,1818 to day 63,2022 & day 63,1920 & $4.85 \times 10^{-4}$ \\
\hline 16.9 & 2 & day 190,0312 to day 190,0517 & day 190,0415 & $6.11 \times 10^{-4}$ \\
\hline 20.0 & 2 & $\ldots$ & day 189,2238 & $3.2 \times 10^{-4}$ \\
\hline 20.9 & 1 & $\ldots$ & day 63,1415 & $2.6 \times 10^{-4}$ \\
\hline
\end{tabular}

*Distance between the spacecraft and the dipole center at the time of the magnetic equatorial plane crossing. Exceptions are 5.3 and $5.9 R_{J}$ where there were no crossings; the listed values are typical distances and times in these 1 wo regions.

$\dagger$ Spectra based on analyzed events are not used at 20.0 and $20.9 R_{J}$ owing to the difference in Voyager 1 and $2 Z>2$ fluxes at these radial distances and beyond (paper 1 ).

$\ddagger$ Magnetic field values from N. F. Ness (private communication, 1980). 
include all magnetic equatorial plane crossings of both spacecraft inside $\sim 20 R_{\boldsymbol{J}}$. In addition, there are two regions at 5.3 and $5.9 R_{J}$ which do not include plane crossings but are at times when Voyager was within $\sim 1 R_{J}$ of the plane. The benefit of calculating spectra near the magnetic equatorial plane will be explained in the next section.

The differential oxygen and sulfur spectra in the $5.3 R_{\mathrm{J}}$ region and the sodium spectrum in the combined 4.9 and $5.3 R_{J}$ regions are shown in Figure 2. The oxygen and sulfur spectra both have a maximum at $\sim 5 \mathrm{MeV} /$ nuc which is most likely due to preferential low-energy losses near lo (paper 1). For energies greater than $\sim 7 \mathrm{MeV} /$ nuc, these two spectra are well represented by power laws of index -6 . The sodium spectrum is softer, with a power law index of -9 . Figure 3 shows the differential oxygen spectra observed in the 7.9 and $10.1 R_{J}$ regions. In these regions the spectra are well represented by a power law of index -7 between 6 and $15 \mathrm{MeV} / \mathrm{nuc}$.

In order to use the $Z>2$ rate point to extend the measured spectrum of a given species to lower energies ( $\sim 1 \mathrm{MeV} /$ nuc), it is necessary to know the fraction of the rate counts that are due to that species. A study was therefore made of the elemental abundances inside $\sim 25 R_{J}$ using analyzed events. The abun-

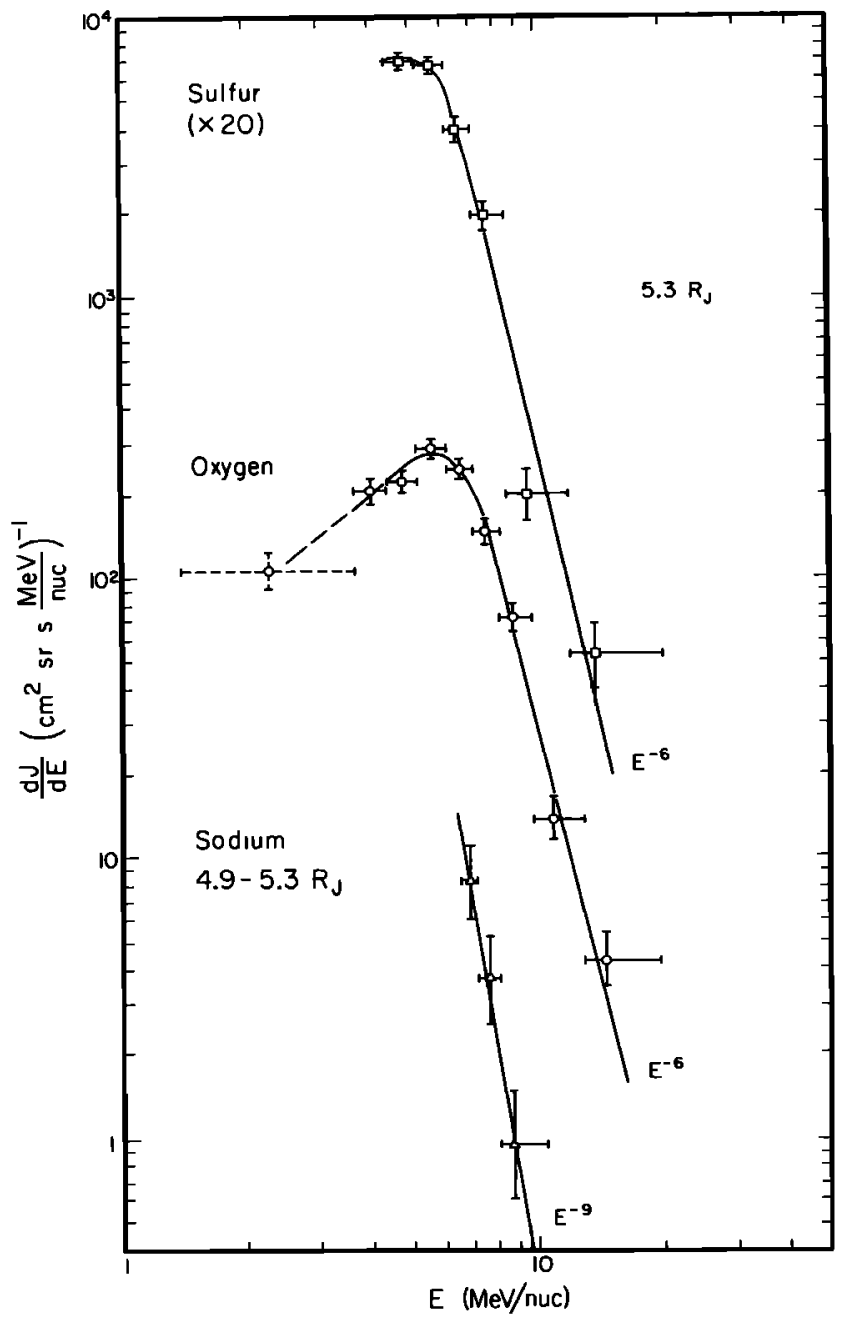

Fig. 2. Differential oxygen, sodium, and sulfur energy spectra near Io. The oxygen and sulfur spectra are from the $5.3 R_{,}$region, and the sodium spectrum is from the combined 4.9 and $5.3 R$, regions (Table 1 ). Note that the sulfur spectrum is multipled by 20 . The oxygen point with dashed error bars at $2.2 \mathrm{MeV} /$ nuc was derived from the $Z>2$ rate.

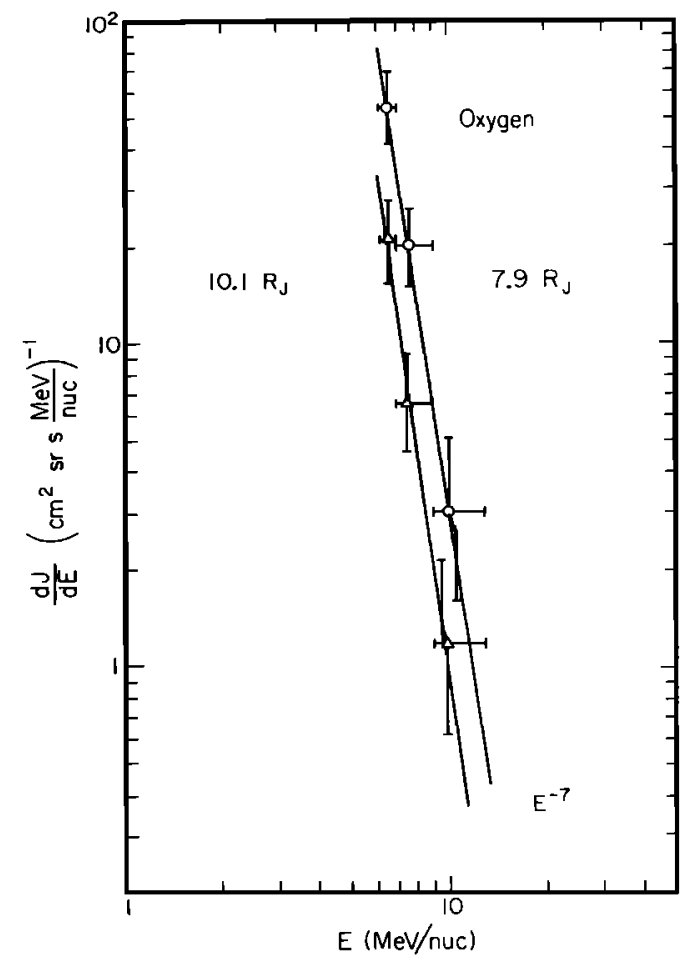

Fig. 3. Differential oxygen spectra from 7.9 and $10.1 R_{\boldsymbol{J}}$.

dance ratios $\mathrm{S} / \mathrm{O}, \mathrm{C} / \mathrm{O}$, and $\mathrm{Na} / \mathrm{O}$ were calculated in several radial ranges by summing events above a given energy-pernucleon threshold. The threshold was then converted to a particle magnetic moment threshold $M=p_{\perp}^{2} / 2 m B$, the first adiabatic invariant, using $M=E / B$, where $E$ is the measured kinetic energy of the ions and $B$ is the measured local magnetic field strength [Ness et al., 1979a, b; N. F. Ness, private communication, 1980]. It is not strictly correct to calculate the magnetic moment thresholds using $M=E / B$ because LET B and $D$ were not pointing exactly perpendicular to the magnetic field direction throughout the region. However, using the actual pointing directions and estimates of the particle pitch angle distributions, we find that, on average, the calculated $M$ is within $\sim 30 \%$ of the real magnetic moment threshold.

The abundance ratios are shown in Figure 4 as a function of $M$. The CRS points are those with horizontal error bars, which represent the range of magnetic field values observed in each radial range. The points with open symbols were calculated using data obtained between 10 and $60 R_{J}$ inbound with the low-energy charged particle (LECP) instrument on Voyager 2 [Hamilton et al., 1981]. The approximate energy ranges for the measurements are 6 to $15 \mathrm{MeV} / \mathrm{nuc}$ for CRS and 0.6 to 1.2 $\mathrm{MeV} /$ nuc for LECP. (The exact energy range for each point is given in the figure caption.) Although the plotted values are not true integral measurements because of the upper energy limits, they are a reasonable representation because the spectra decrease rapidly with energy.

The agreement between the CRS and LECP data in Figure 4 indicates that the relative abundances of carbon, oxygen, sodium, and sulfur at a given magnetic moment are approximately the same throughout the dayside magnetosphere. The lines shown in the figure are power law fits to the combined data sets. $\mathrm{S} / \mathrm{O}$ and $\mathrm{Na} / \mathrm{O}$ are seen to increase toward smaller magnetic moments while $\mathrm{C} / \mathrm{O}$ decreases. Therefore an instrument measuring abundance ratios in a fixed energy range will detect increasing sulfur and sodium and decreasing carbon 


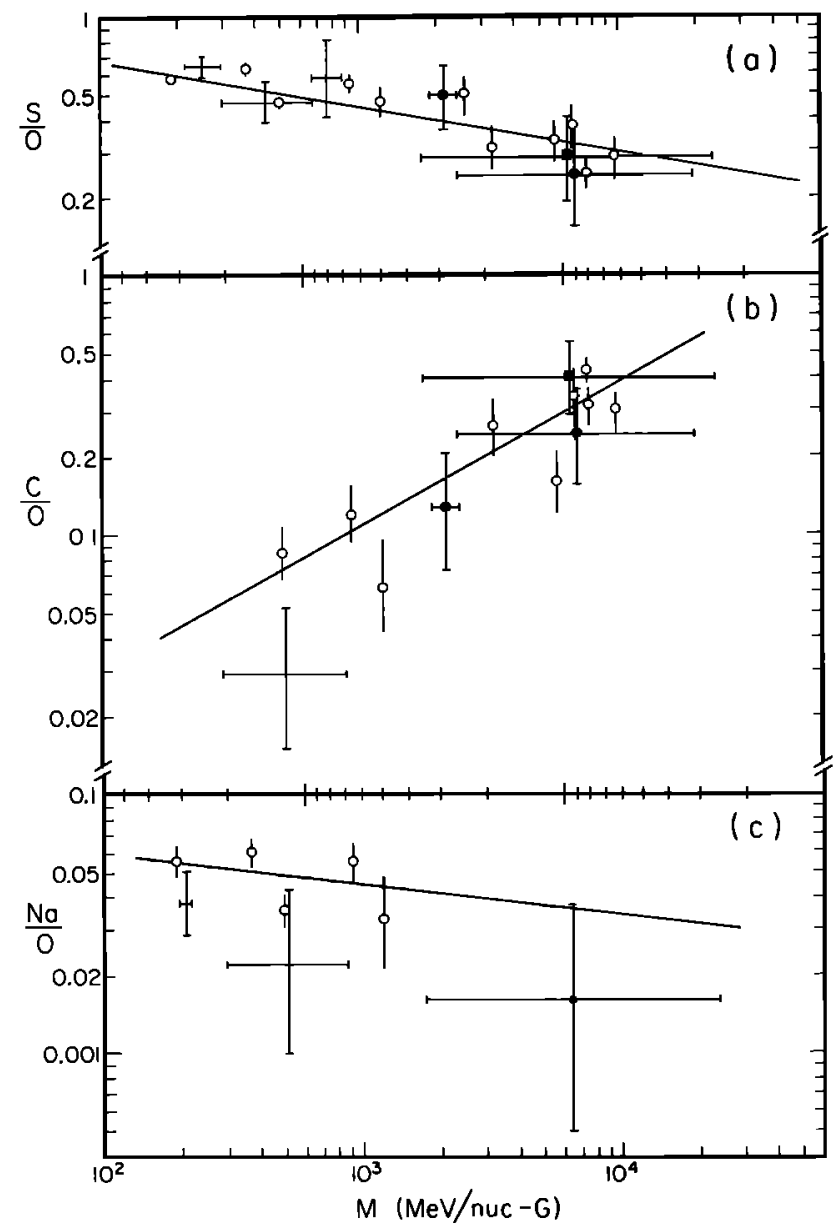

Fig. 4. Abundance ratios with respect to oxygen of $(a)$ sulfur, $(b)$ carbon, and $(c)$ sodium as functions of the particle magnetic moments. The open symbols were calculated using published LECP data [Hamilton et al., 1981]. The symbols in the plot are defined as follows: plain cross, Voyager 1 6.6-17.3 MeV/nuc; solid circle, Voyager 2 7.5-11.0 $\mathrm{MeV} / \mathrm{nuc}$; solid square, Voyager 1 4.7-14.8 MeV/nuc; solid diamond, sum of solid circle and solid square; open circle, LECP Voyager 2 $0.60-1.15 \mathrm{MeV} /$ nuc. The vertical error bars represent statistical uncertainties. The lines are power law fits to the combined CRS and LECP data.

abundances relative to oxygen as it moves toward smaller radial distances (smaller $M$ ). Also, an instrument at a fixed radial position will measure a sulfur to oxygen and sodium to oxygen ratio that increases toward lower energies and a carbon to oxygen ratio that decreases, since the sulfur and sodium spectra are softer than the oxygen spectrum and the carbon spectrum is harder. If the approximation is made that the energy spectra of these elements are proportional to $E^{-\gamma}$, then the fits shown in the figure indicate that $\gamma_{s}=\gamma_{o}+0.2, \gamma_{c}=\gamma_{o}$ -0.6 , and $\gamma_{\mathrm{Na}}=\gamma_{\mathrm{o}}+0.1$. The harder carbon spectrum may reflect a solar wind origin for carbon [Hamilton et al., 1981], whereas the other species are Iogenic.

In the next section, oxygen integral spectra are calculated for each of the regions in Table 1 and are used to determine the radial profile of the oxygen phase space density inside $\sim 20 R_{J}$. The data in Figure 4 will be used to calculate the oxygen fraction of the $Z>2$ rate so that the spectra can be extended down to $\sim 1 \mathrm{MeV} /$ nuc.

\section{Phase Space Density Determination}

In order to compare spectral measurements made at different locations in a magnetosphere, it is useful to relate them to a diffusion theory. If it is assumed that particles conserve their first and second adiabatic invariants in the diffusion process, but violate their third invariant (see, e.g., Schulz and Lanzerotti [1974]), then the radial diffusion equation is

$$
\frac{\partial f}{\partial t}=L^{2} \frac{\partial}{\partial L}\left[\frac{D}{L^{2}} \frac{\partial f}{\partial L}\right]+S(L)-\mathfrak{L}(L)
$$

where $f$ is the phase space density at constant first adiabatic invariant $(M), D$ is the diffusion coefficient, $L$ is the Mcllwain parameter, and $\delta(L)$ and $L(L)$ represent local sources and sinks, respectively.

The relationship between the integral particle flux $J_{\perp}$ and the phase space density is given by

$$
f\left(M, L, \alpha=90^{\circ}\right)=\frac{\gamma(M, L)}{2 m M^{2}} \frac{J_{\perp}(M, L)}{B^{2}(L)}
$$

(paper 1), where $\alpha$ is the particle pitch angle and $\gamma=-[d \log$ $\left.\left(J_{1}\right)\right] /[d \log (M)]$. Equation (2) shows that in order to calculate $f$, one must measure the integral flux perpendicular to the magnetic field direction (i.e., the flux of mirroring particles). Also, implicit in the diffusion equation is the requirement that $f$ be determined along the dynamic path that particles follow in the diffusion process. To satisfy these two requirements, we have restricted analysis to ions mirroring at the magnetic equatorial plane. Since LET B and D were not in general oriented perpendicular to the field lines at the time of the plane crossing, the mirroring flux $J_{\perp}$ was calculated from the measured fluxes by applying a pointing correction factor. The correction is based on estimates made of the pitch angle distribution of the energetic heavy ions inside $20 R_{J}$. The telescope pointing directions and the pointing correction factor in each region are listed in Table 2. Also listed are the live time correction factors for the $Z>2$ rate, the analyzed event rate as determined by laboratory measurements, and the effective energy thresholds of the $Z>2$ rate for oxygen ions (paper 1 ).

The oxygen fraction of the $Z>2$ rate was also used to calculate $J_{\perp}$. This number was determined from the data in Figure 4 and, owing to the offsetting effects of a decreasing carbon abundance and an increasing sulfur abundance toward smaller radial distances, is approximately constant and equal to $1 / 2$ throughout the region between 6 and $17 R_{J}$. Note that this number is not an oxygen abundance fraction, since the energy threshold of the $Z>2$ rate (in energy per nucleon) is different for different elements.

The quantity $J_{1} / B^{2}$, used in calculating the phase space density (equation (2)), is shown as a function of $M$ in Figure 5 for the regions listed in Table 1. The magnetic field strength for each region (Table 1) is the value that was measured at the time of the magnetic equatorial plane crossing. The continuous steplike part of each spectrum is based on analyzed events, and the solid symbol with an error bar is calculated from the $Z>2$ rate. The long-dashed curves joining the two is an interpolation. The event portions of the Voyager 113.0 and $16.8 R_{J}$ spectra are represented by single points (open circles) instead of by complete spectra owing to the few events obtained in these regions. These two measurements, as well as the $Z>2$ flux measurements in the two regions, are important, because they show that to within a factor of 2 , the Voyager 1 and 2 fluxes of energetic oxygen ions were the same between 12 and $17 R_{J}$.

The short-dashed and dash-dotted curves in Figure 5 are extrapolations of the measured spectra to smaller magnetic moments. The error bars associated with these parts of the 
TABLE 2. Quantities Used to Calculate $J_{\perp}$

\begin{tabular}{|c|c|c|c|c|c|c|}
\hline$\underset{R_{J}}{\text { Region, }}$ & LET* & $\begin{array}{l}\text { Pointing Angle } \\
\text { With Respect to } \\
\text { Field Line, deg }\end{array}$ & $\begin{array}{l}\text { Pointing } \\
\text { Correction } \\
\text { Factort }\end{array}$ & $\begin{array}{c}Z>2 \text { Live Time } \\
\text { Correction } \\
\text { Factor } \neq\end{array}$ & $\begin{array}{l}\text { Event Live Time } \\
\text { Correction } \\
\text { Factor }\end{array}$ & $\begin{array}{c}\text { Effective Oxygen } \\
Z>2 \text { Threshold, } \$ \\
\text { MeV/nuc }\end{array}$ \\
\hline 4.9 & B & 68 & 1.1 & 1.3 & 1.5 & 1.4 \\
\hline 5.3 & B & 62 & 1.2 & 1.2 & 1.5 & 1.4 \\
\hline 5.9 & B & 60 & 1.3 & 1.3 & 1.5 & 1.5 \\
\hline \multirow[t]{2}{*}{7.9} & B & 55 & 1.3 & 1.7 & 1.5 & \\
\hline & D & 53 & 1.4 & 1.6 & 1.5 & 1.5 \\
\hline 10.1 & B & 65 & 1.2 & 1.4 & 1.6 & 1.3 \\
\hline \multirow[t]{2}{*}{12.2} & B & 77 & 1.1 & 1.2 & 1.6 & \\
\hline & D & 41 & 1.6 & 1.2 & $\begin{array}{l}1.0 \\
1.6\end{array}$ & 1.2 \\
\hline \multirow[t]{2}{*}{13.0} & B & 78 & 1.1 & 1.1 & 1.6 & \\
\hline & D & 36 & 1.8 & 1.1 & 1.6 & 1.1 \\
\hline \multirow[t]{2}{*}{13.7} & B & 57 & 1.2 & 1.1 & 1.6 & \\
\hline & D & 44 & 1.6 & 1.1 & 1.6 & 1.1 \\
\hline \multirow[t]{2}{*}{16.8} & B & 49 & 1.4 & 1.0 & 1.6 & \\
\hline & D & 50 & 1.4 & 1.0 & 1.6 & 1.1 \\
\hline \multirow[t]{2}{*}{16.9} & B & 75 & 1.1 & 1.0 & 1.6 & \\
\hline & D & 53 & 1.4 & 1.0 & 1.6 & 1.1 \\
\hline \multirow[t]{2}{*}{20.0} & $\bar{B}$ & 71 & 1.1 & 1.0 & $\ldots$ & \\
\hline & D & 42 & 1.6 & 1.0 & $\ldots$ & 1.1 \\
\hline \multirow[t]{2}{*}{20.9} & B & 90 & 1.1 & 1.0 & $\ldots$ & \\
\hline & D & 48 & 1.5 & 1.0 & $\ldots$ & 1.1 \\
\hline
\end{tabular}

*In the $4.9,5.3,5.9$, and $10.1 R_{J}$ regions, only LET B was used because it provided adequate statistics by'itself and was more nearly perpendicular to the magnetic field than LET $D$.

tA Compton-Getting correction of magnitude $\$ 0.05$ is included in these factors. The correction factors for 5.3 and $5.9 R_{J}$ take into account the nonzero magnetic latitude of the spacecraft in these regions.

$\$ 1.0$ implies no correction.

ๆThe 4.9, 5.3, and 5.9 $R_{J}$ spectra have an additional pulse pileup correction that has been applied to the event portions, of magnitude 1.4 for $E \lesssim 7 \mathrm{MeV} / \mathrm{nuc}$ (paper 1).

$\S$ The nominal oxygen threshold is $1.1 \mathrm{MeV} /$ nuc (sulfur is $0.9 \mathrm{MeV} /$ nuc, carbon is $1.2 \mathrm{MeV} / \mathrm{nuc}$ ). These thresholds include the particle energy loss in the telescope window.

spectra represent the uncertainty in the extrapolations. Except at $10.1 R_{J}$, the upper and lower limits for each error bar were determined by extending the spectra from the $Z>2$ point with lines of slope $\gamma_{z>2}$ and $\gamma_{z>2} / 1.4$, respectively, where $\gamma_{z>2}$ is the slope at the $Z>2$ point (see Figure 6). The $\gamma_{Z>2}$ and $\gamma_{Z>2} / 1.4$ extrapolation limits were chosen as reasonable estimates based on the LECP spectral shape and observed fluxes of 0.6-1.15 $\mathrm{MeV} /$ nuc-G oxygen ( $\sim 250 \mathrm{MeV} /$ nuc-G at $11 R_{J}$ ). At $10.1 R_{,}$a longer extrapolation was performed than in other regions in order to extend the spectrum to $70 \mathrm{MeV} /$ nuc-G. In this case, the shape of the spectrum was calculated using the energy-percharge spectrum measured at these magnetic moments by LECP at $\sim 16 R_{J}$ on Voyager 2 [Hamilton et al., 1981].

LECP observations were also used as a consistency check on the slopes of the spectra at the $Z>2$ points. Figure 6 shows these slopes $\left(\beta=\gamma_{Z>2}+1\right)$ compared with those measured in the energy range from 0.60 to $3.17 \mathrm{MeV} /$ nuc between 10 and 25 $R_{J}$ by LECP [Hamilton et al., 1981]. The values from the two instruments are the same within statistics, and both show the same trend of softer spectra toward increasing radial distance.

The oxygen phase space density at several values of $M$, calculated using (2) and the spectra in Figure 5, is shown in Figure 7 as a function of $L$. Since these measurements were obtained near the magnetic equatorial plane, $L$ is defined to be the spacecraft-dipole center distance at the time of the plane crossing. The uncertainties shown for each measurement represent uncertainties in the magnetic field strength $(\sim 3 \%)$, in the oxygen fraction of the $Z>2$ rate $(\sim 15 \%)$, in $\gamma(M, L)(\sim 15 \%)$, and in the pointing correction factor $(\sim 10 \%)$ and statistical counting uncertainties in the number of events in the event portions of the spectra. An additional $\sim 15 \%$ systematic uncertainty in the live time correction factor applies to all measure- ments. Inside $7.9 R_{J}$ the lines connecting points of the same magnetic moment are dotted to indicate that there may be discontinuities in the shape of the phase space density profile in this region due to discontinuities in the diffusion coefficient [Siscoe et al., 1981].

As was previously observed [Vogt et al., 1979b; paper 1], there is a positive radial gradient in the phase space density of the energetic oxygen ions between 5.6 and $17 R_{J}$, indicating an inward diffusive flow in this region. The objective in the next sections will be to estimate the diffusion coefficient in the region and to determine the rate at which ions and energy flow into the inner magnetosphere.

\section{Limits ON THE Diffusion CoEfFicient and Mass Loading Rate}

The measured radial dependence of the oxygen phase space density (Figure 7) can now be used to obtain solutions to the diffusion equation and thereby set limits on the diffusion coefficient. The diffusion equation (1) for a steady state condition in a region in which there are no sources and the loss term is of the form $\mathcal{L}(L)=f / \tau$, where $\tau$ is the particle lifetime, is

$$
L^{2} \frac{\partial}{\partial L}\left[\frac{D}{L^{2}} \frac{\partial f}{\partial L}\right]=\frac{f}{\tau}
$$

In this analysis the phase space density profile at a given magnetic moment is approximated as a series of power law lines $\left(f \propto L^{q}\right)$ connecting the measurements. Comparison with the smoothed curves in Figure 7 indicates that this is a reasonable approximation. Table 3 lists the values of the power law indices $q$ that will be used. 


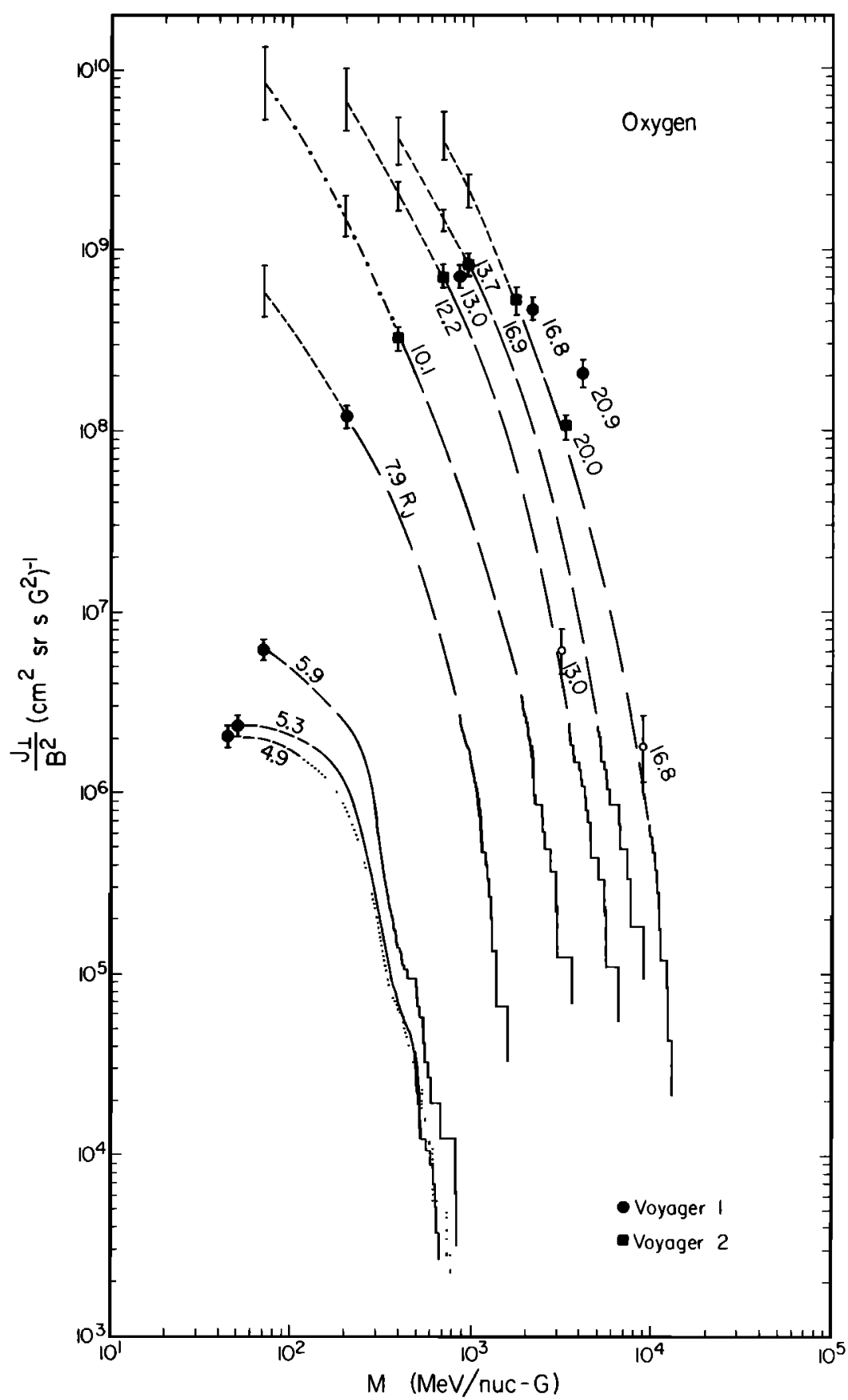

Fig. 5. Integral oxygen spectra from all regions listed in Table 1 divided by the square of the local magnetic field strengths, as functions of magnetic moment. The solid line portion of each spectrum is based on pulse-height-analyzed

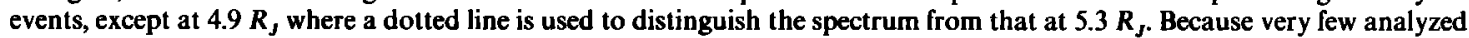
events were observed by Voyager 1 at 13.0 and $16.8 R_{b}$, those data are represented by open circles with error bars rather than by integral spectra. The solid symbols which indicate the integral fluxes obtained from the $Z>2$ rate are connected by long-dashed lines to the corresponding spectrum of analyzed events observed at 4.9, 5.3, 5.9, 7.9, 10.1, 12.2, 13.7, and $16.9 R_{J}$. Additional $Z>2$ fluxes observed at 13.0,16.8, 20.0. and $20.9 R_{J}$ are shown for comparison. The short-dashed curves and the dash-dotted curve represent extrapolations based on data from the Low-Energy Charged Particle Experiment as discussed in the text. The error bars at the lower limit of the extrapolations indicate the uncertainties in the extrapolations. Note that these are not true integral spectra, since oxygen nuclei with $\gtrsim 23 \mathrm{MeV} /$ nuc are not analyzed [Gehrels et al., 1981]. Thus there is an unmeasured additive contribution to these spectra. For example, if the differential spectrum in Figure 2 at $5.3 R_{J}$ extends to higher energies as $E^{-6}$, the additional contribution would be $\sim 10^{3} \mathrm{~cm}^{-2} \mathrm{~s}^{-1} \mathrm{sr}^{-1} \mathrm{G}^{-2}$.

We first consider the case of no losses $(\tau \rightarrow \infty)$ between 6 and $17 R_{J}$. In this limit,

$$
D \propto L^{3-q} s^{-1}
$$

Between 5.9 and $12.2 R_{J}, q$ is greater than 8 (Table 3), so that the diffusion coefficient would have to have an index $n(D \propto L)$ of less than -5 to give the observed radial dependence of the oxygen phase space density if there are no losses in the region. A large negative index such as this is inconsistent with other determinations of $\boldsymbol{n}$ made using observations of energetic electrons and protons and of plasma in the region [Thomsen et al., 1977; Goertz et al., 1979; Froidevaux, 1980; Siscoe et al., 1981], 


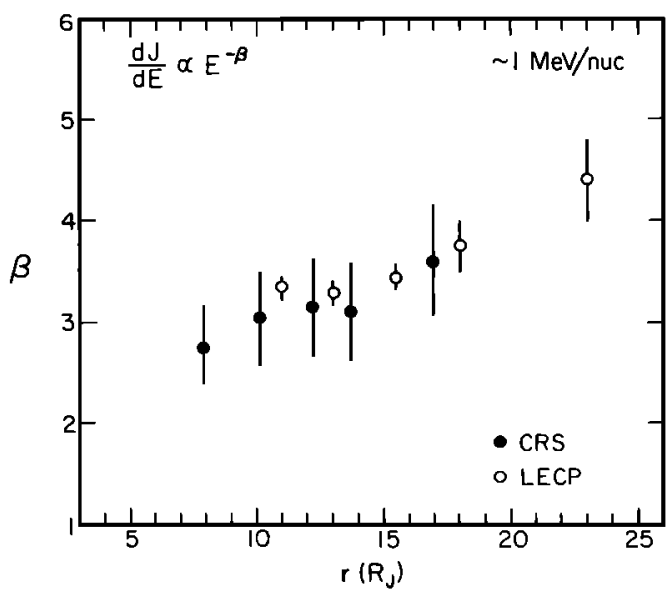

Fig. 6. A comparison of the logarithmic slope of the oxygen spectrum at the $Z>2$ point $(\sim 1 \mathrm{MeV} /$ nuc) observed by CRS and the power law index between 0.60 and $3.17 \mathrm{MeV} /$ nuc measured by LECP [Hamilton et al., 1981]. Note that $\beta$ is the power law index for a differential spectrum ( $\left.d J / d E \propto E^{-\beta}\right)$, so that for the CRS observations, $\beta=\gamma_{z>2}+1$, where $\gamma_{z>2}$ is the slope of the integral spectrum at the $Z>2$ point.

which can be summarized as $n=4 \pm 2$. We therefore conclude that there must be losses between 6 and $12 R_{J}$, and hence we will now consider the case of lossy diffusion.

It will be assumed that the losses are occurring in the strong pitch angle diffusion limit. In this limit, there is sufficient pitch angle scattering to refill the loss cone with particles as quickly as it empties, so that the lifetime of the particles to pitch angle scattering is a minimum. The loss cone solid angle at the magnetic equatorial plane (both directions) is approximately $2 \pi \alpha_{0 l}{ }^{2}$, where $\alpha_{0 l}$ is the equatorial loss cone angle, and the time required to empty it of particles is approximately one quarter of the bounce period $\tau_{b}$ [Kennel and Petschek, 1966]. Therefore for nonrelativistic particles at constant $M$, the strong pitch angle diffusion lifetime $\tau_{s}$, assuming a dipolar Jovian magnetic field of $4 \mathrm{G} R_{J}{ }^{3}$, is given by

$$
\tau_{s}=\frac{\tau_{b} / 4}{\alpha_{0 l}{ }^{2} / 2} \approx 5 M^{-1 / 2} L^{11 / 2}[4-3 / L]^{1 / 2} \mathrm{~s}
$$

where $[M]=\mathrm{MeV} /$ nuc-G. The deviation of the measured magnetic field from a dipole [Connerney et al., 1981] tends to decrease $\alpha_{0 l}$ and thereby increase $\tau_{s}$. The difference, however, is estimated to be less than $\sim 50 \%$ between 6 and $17 R_{J}$, and in the analysis that follows, (5) will be used to calculate $\tau_{s}$.

Using $\tau=\tau_{s}$ in the diffusion equation (3) and assuming $f \propto L^{q}$ and $D \propto L^{n}$, the upper limit for the diffusion coeflicient $D_{\mathrm{u}}$ is

$$
D_{u}(L, M)=\frac{L^{2}}{q(q+n-3) \tau_{s}(L, M)} \mathrm{s}^{-1}
$$

This represents an upper limit because it is based on the minimum particle lifetime $\tau_{s}$.

In a recent analysis of Voyager plasma data [Siscoe et al., 1981], the diffusion coefficient between 8 and $9 R_{J}$ was found to be of the form $D=2.6 \times 10^{-37} S_{i} L^{4} \mathrm{~s}^{-1}$, where $S_{i}$ (in ions per second) is the ion source strength from Io, also called the mass loading rate. As discussed in the introduction, previous studies indicate that $S_{i}$ is most likely in the range from $10^{27}$ to $6 \times 10^{28}$ ions/s, with a nominal value of $6 \times 10^{27}$ ions/s. Thus the magnitude of the diffusion coefficient can only be estimated from the plasma analysis. The radial dependence of $D\left(D \propto L^{4}\right)$ is, however, directly determined from the measured radial depen- dence of the plasma density in the region, and we will therefore use $n=4$ in (6) between 8 and $9 R_{J}$.

The diffusion coefficient upper limit calculated at $L=8.9$ with (6) using $n=4, q$ from Table 3, and $\tau_{s}$ from (5) is shown for several magnetic moments in Figure 8 . These limits are not very sensitive to the value used for $\boldsymbol{n}$ as is indicated by the dashed error bar which represents the range of $D_{u}$ for $9.4 \times 10^{2}$ $\mathrm{MeV} / \mathrm{nuc}-\mathrm{G}$ as $n$ varies between 0 and 12 . The solid lines between 8 and $9 R_{J}$ in Figure 8 are the plasma diffusion coefficient for $S_{i}=10^{27}, 10^{28}$, and $10^{29}$ ions/s. It is seen that the upper limits imposed on the diffusion coeflicient at $8.9 R_{J}$ by the energetic oxygen observations, when combined with the plasma analysis of Siscoe et al. [1981], place an upper limit on the mass loading rate of $\sim 10^{28}$ ions/s.

At radial distances larger than $9 R_{J}$, the diffusion coefficient upper limits were obtained from the value at $8.9 R_{J}$ using a finite difference solution of the diffusion equation (3). For example, $D\left(11.4 R_{J}\right)$ was calculated using

$$
\begin{array}{r}
10.1^{2}\left(\frac{D(11.4)(\partial f / \partial L)(11.4) / 11.4^{2}-D(8.9)(\partial f / \partial L)(8.9) / 8.9^{2}}{11.4-8.9}\right) \\
=\frac{f(10.1)}{\tau_{s}(10.1)}
\end{array}
$$

The results are shown in Figure 8. The diffusion coefficient upper limit that will be used in the analysis that follows is represented by the dashed line labeled $D_{u}$, which is a fit to the measurements. The radial dependence of this fit between 9 and $17 R_{J}$ is $D_{u} \propto L^{1.6}$. The reasonable lower limit of $\sim 10^{27}$ ions $/ \mathrm{s}$ for $S_{i}$ (see the introduction) was used to determine a lower limit for the diffusion coefficient, labeled $D_{1}$ in the figure. The fact that $D_{l}$ is less than a factor of 10 below $D_{u}$ implies that the particle lifetime is within an order of magnitude of the strong pitch angle diffusion lifetime in this region. The limits shown at $5.3 R_{J}$ were not calculated from the energetic oxygen data but represent the range of values determined from energetic proton and electron measurements [Thomsen et al., 1977; Goertz et al., 1979].

Fits performed on previously published CRS and LECP phase space density measurements in the inner magnetosphere [Thorne, 1982] give upper limits to the diffusion coefficient that are similar to those shown in Figure 8. For instance, at $9 \boldsymbol{R}_{J}$ Thorne finds $D_{u}$ to be in the range $8 \times 10^{-6}$ to $3 \times 10^{-5} \mathrm{~s}^{-1}$ compared with $\sim 10^{-5} \mathrm{~s}^{-1}$ shown in the figure. Note, however, that Thorne's model fits assumed that $D \sim L^{n}$ throughout the region, so that the resulting radial dependence differs from that in Figure 8.

Although diffusion coefficient limits between 5.3 and $8 R_{J}$ are not needed in the analysis that follows, the radial dependence of $D$ in this region, as determined from the plasma data [Siscoe et al., 1981] (assuming $S_{i}=10^{28}$ ions/s), is also displayed in Figure 8. The discontinuities in $D$ are due to observed discontinuities in the radial dependence of the plasma density.

\section{Particle and Energy Flow Rates}

The diffusion coefficient limits shown in Figure 8 will now be used in combination with the measured phase space densities and energy spectra to determine the inward diffusive flow rate of the energetic oxygen ions. The diffusive flow rate of ions with magnetic moments greater than $M, F(>M)$ across radius $r$ is given by

$$
F(>M)=n(>M) \cdot 2 \pi r \cdot 2 h \cdot v_{D} \cdot R_{J}^{3} \text { ions } / \mathrm{s}
$$




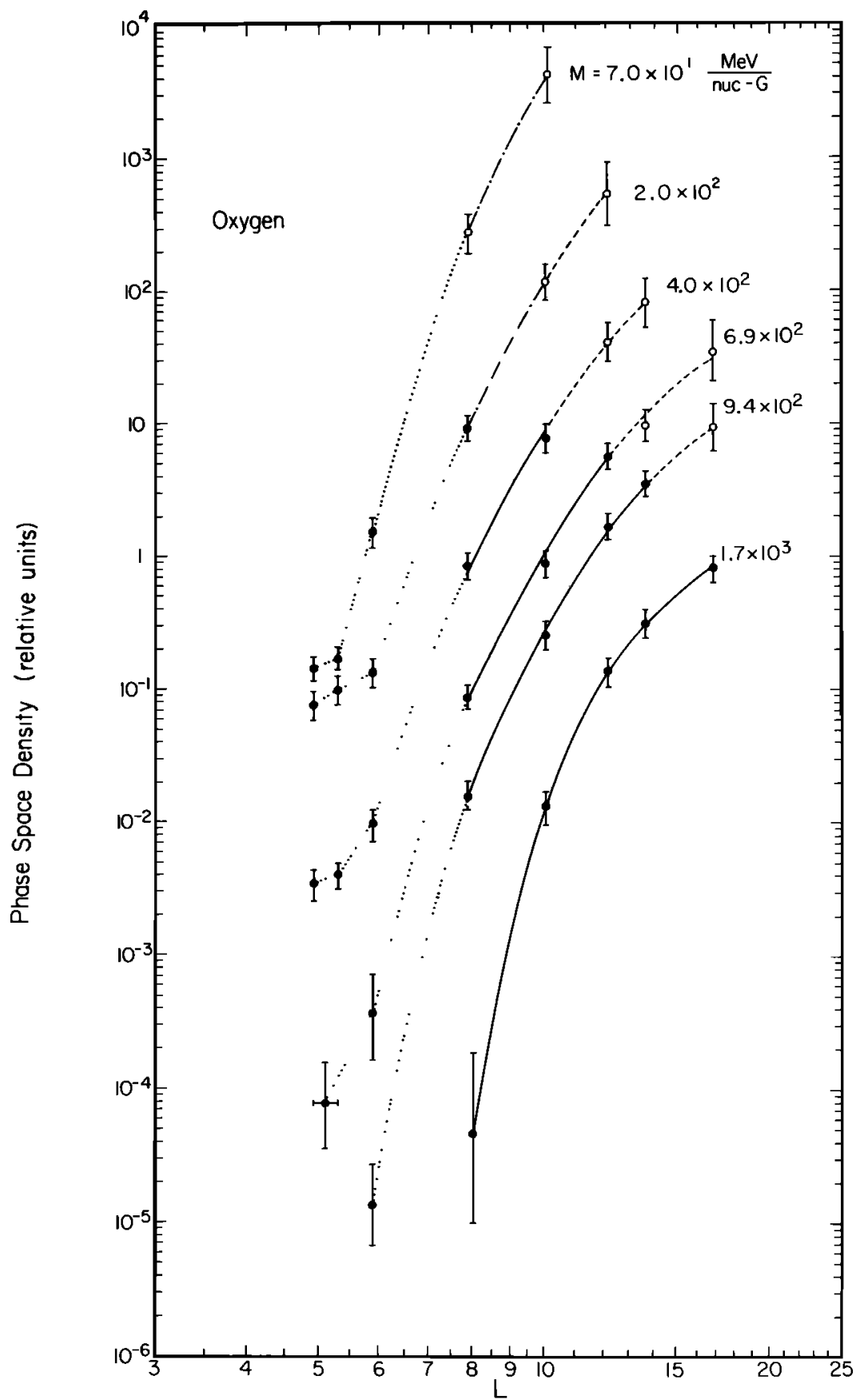

Fig. 7. The radial dependence of the phase space density of the energetic oxygen ions al constant magnetic moment, for several values of $M$. The solid points are derived from the measured portions of the spectra shown in Figure 5, while the open points ate based on the extrapolations. The 4.9 and $5.3 R_{J}$ measurements at $6.9 \times 10^{2} \mathrm{MeV} /$ nuc-G were averaged together to improve statistics. Solid lines join the measurements at given magnetic moments between 7.9 and $16.9 R_{J}$. The 5.3, 5.9, and $7.9 R$, measurements are connected by dotted lines to indicate that the radial profiles in this region may have discontinuities between the points.

where $n(>M)$ is the number density $\left(\mathrm{cm}^{-3}\right)$ of oxygen ions with magnetic moment greater than $M$ calculated from the spectra, $h$ is the scale height $\left(R_{J}\right)$ of the particle distribution relative to the magnetic equatorial plane, and $v_{D}$ is the average radial diffusion velocity $\left(R_{J} / \mathrm{s}\right)$ given by $v_{D}=D(1 / f)(\partial f / \partial L)$. (see, e.g., Schulz and Lanzerotti [1974]). Based on measurements of the latitudinal dependence of the energetic $Z>2$ fluxes and on estimates of the particle pitch angle distributions, $h$ was found to be $\sim 3 \boldsymbol{R}_{\boldsymbol{J}}$.

Figure $9 b$ shows the radial profile of $F(>M)$ for several magnetic moment thresholds, assuming $D(L)$, which is the geometric mean of $D_{u}$ and $D_{l}\left(\right.$ e.g., $\left.D\left(9 R_{J}\right) \approx 4 \times 10^{-6} \mathrm{~s}^{-1}\right)$. Note that if the actual diffusion coefficient is a factor of 3 larger (or smaller), $F(>M)$ will be correspondingly larger (or smaller). The typical uncertainty in the calculated points represents uncertainties in $n(>M)(\sim 20 \%), h(\sim 20 \%),(1 / f)(\partial f / \partial L)(\sim 15 \%)$, and $D(\sim 50 \%)$. The inward flow rate of $>400 \mathrm{MeV} /$ nuc-G oxygen ions at $10 R_{J}$ is $\sim 10^{22}$ ions $\mathrm{s}^{-1}$, somewhat larger than, but consistent with, the less accurate estimate in paper 1 of $5 \times 10^{21 \pm 1}$ ions $\mathrm{s}^{-1}$. The inward flow rate at all magnetic moments is approximately constant between 17 and $12 R_{J}$, implying that few particles are lost in this region. Between 12 and $6 R_{J}$, on the other hand, the rate steeply decreases so that, 
TABLE 3. Power Law Indices of the Oxygen Phase Space Density

\begin{tabular}{cccccc}
\hline & \multicolumn{5}{c}{$q$} \\
\cline { 2 - 6 }$L_{1}-L_{2}$ & $M=200^{*}$ & $M=400$ & $M=690$ & $M=940$ & $M=1700$ \\
\hline $5.9-7.9$ & 14.5 & 15.4 & 18.8 & 24.2 & $\ldots$ \\
$7.9-10.1$ & 10.4 & 9.0 & 9.4 & 11.4 & $\ldots$ \\
$10.1-12.2$ & 8.0 & 8.8 & 9.9 & 9.9 & 12.3 \\
$12.2-13.7$ & $\cdots$ & 5.9 & 4.5 & 6.4 & 7.2 \\
$13.7-16.9$ & $\cdots$ & $\cdots$ & 6.1 & 4.7 & 4.6 \\
\hline
\end{tabular}

Calculated using the data shown in Figure 7.

"Units of MeV/nuc-G.

for example, 6 times more particles with $M>200 \mathrm{MeV} /$ nuc-G are flowing inward at $10 R_{J}$ than at $8 R_{J}$. Since we are assuming an equilibrium condition in which the density of particles between 10 and $8 R_{J}$ is not increasing, the difference in the inward flow rates is a direct indication of large particle losses. The

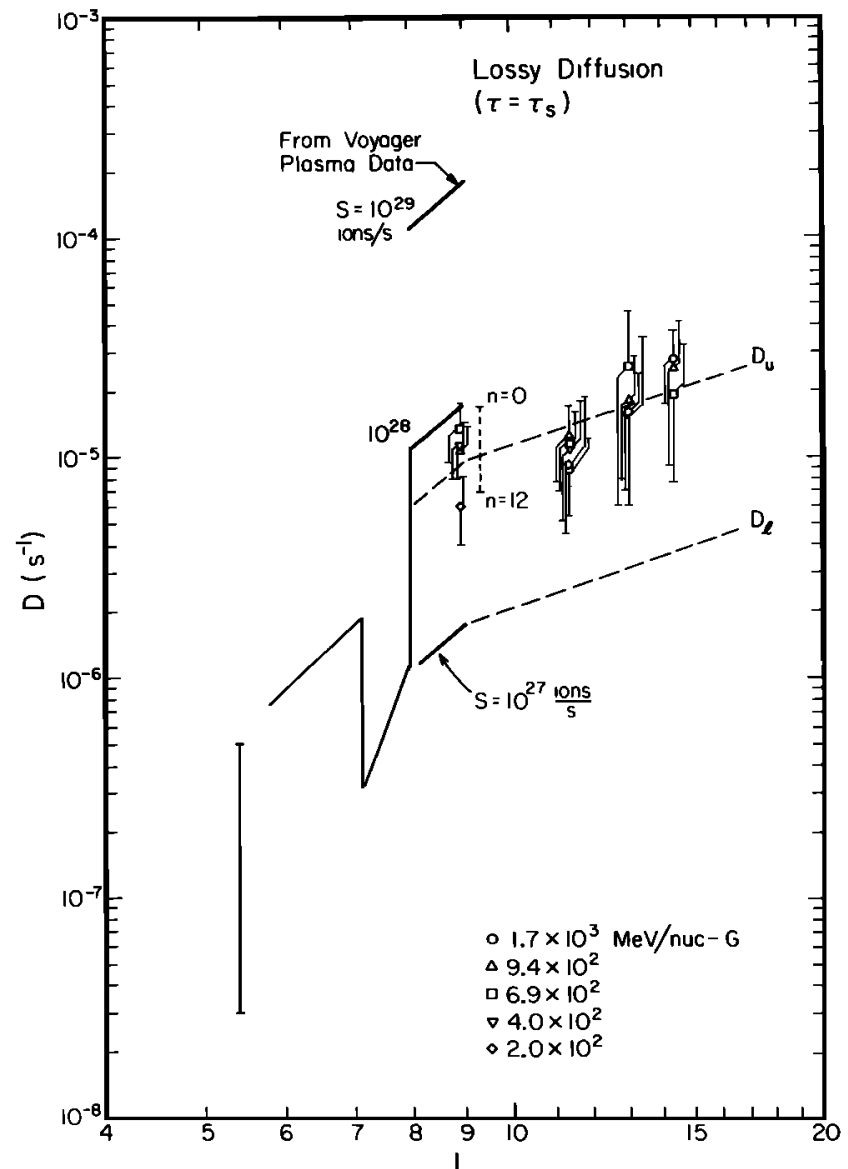

Fig. 8. Diffusion coefficient limits between 5.3 and $17 R_{J}$. The

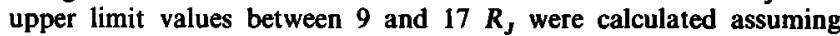
strong pitch angle diffusion ( $\tau=\tau_{s}, \tau_{s}$ being given by (5)). The points at $8.9 R_{J}$ were determined using (6) with $n=4$ as indicated by Voyager plasma data between 8 and $9 R_{J}$ [Siscoe et al., 1981]. The dashed error bar represents the range of values that the $9.4 \times 10^{2} \mathrm{MeV} /$ nuc-G point assumes as $n$ varies from 0 to 12 . The solid lines in the portion of the figure between 8 and $9 R$, show the dependence of the plasma diffusion coefficient on the Io source strength $S$ of oxygen and sulfur ions. The points at 11.4, 13.0, and 14.4 $R_{J}$ were calculated using equations like (7). The dashed lines are the diffusion coefficient limits $\left(D_{u}\right.$, upper; $D_{l}$ lower) that will be used in the analysis that follows, with the range at $5.3 R_{I}$ determined from Pioneer measurements of energetic protons and electrons [Thomsen et al., 1977; Goertz et al., 1979]. The diffusion coefficient shown between 5.8 and $8 R_{J}$ is that obtained from the plasma analysis [Siscoe et al., 1981] assuming $S_{i}=10^{2 \mathrm{~B}}$ ions/s. region in which the largest total number of energetic oxygen ions is lost is between 8 and $12 R_{J}$.

The likely cause of the losses is pitch angle scattering into the loss cone, as can be seen by the following arguments (see also Thorne [1982]). First, a comparison with the plasma charge density in Figure 9a [Bagenal and Sullivan, 1981] shows that the region where most of the ions are lost $\left(8-12 R_{J}\right)$ is outside the region of maximum plasma density and well outside the orbit of Io (5.9 $\left.R_{J}\right)$. Hence geometric absorption by Io and energy loss in the plasma torus near Io are ruled out as the dominant loss mechanisms. Second, the fact that few particles are lost outside $\sim 12 R_{J}$ and that large losses occur inside $\sim 10$

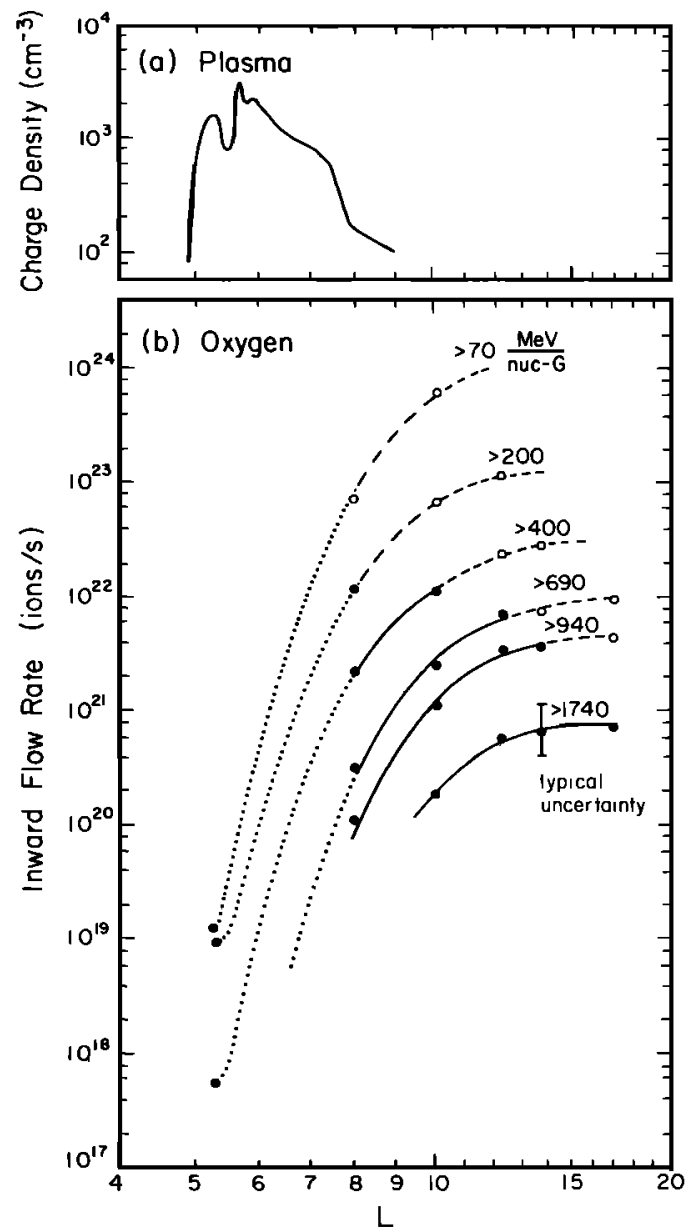

Fig. 9. (a) The plasma charge density near Io [Bagenal and Sullivan, 1981]. (b) The inward flow rate of oxygen ions with magnetic moments greater than the indicated values. The points were calculated using (8). A typical error bar is shown. There is, in addition, a factor of 3 absolute uncertainty that applies to all points. Smoothed curves are drawn through points of given magnetic moment threshold. 


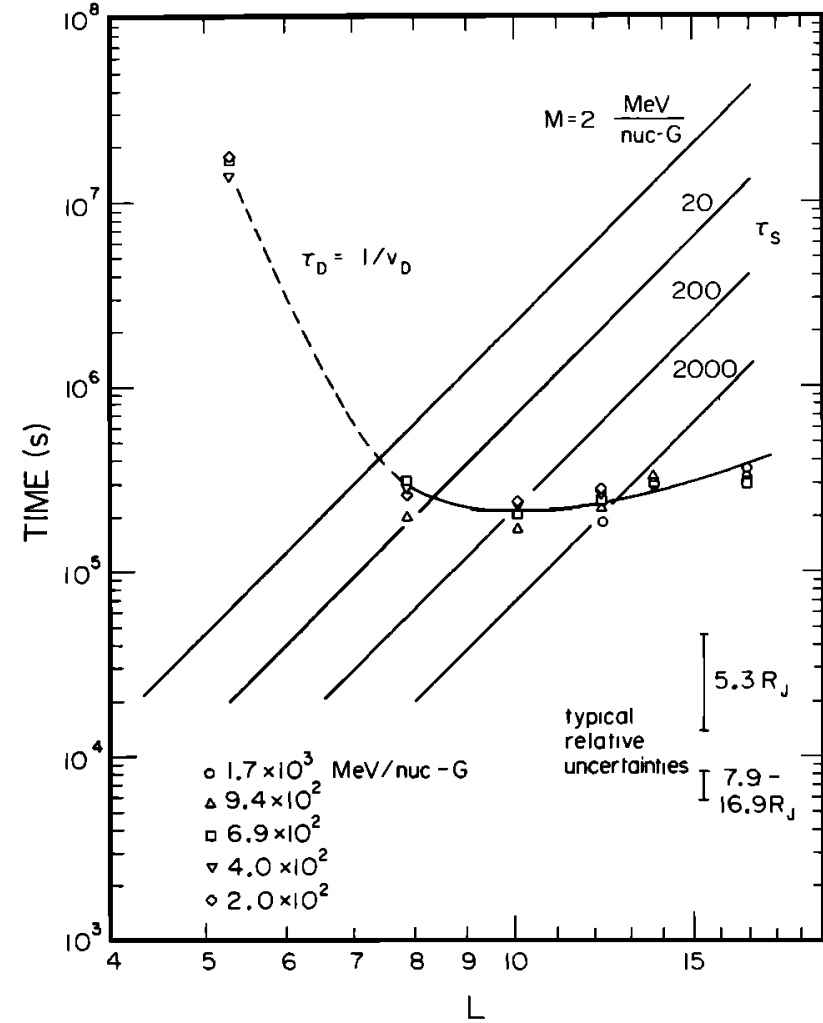

Fig. 10. Comparison of the diffusion time $\tau_{D}=1 / v_{D}$, where $v_{D}=$ $D(1 / f)(\partial f / \partial L)$, with the particle lifetime $\tau_{s}$ in the strong pitch angle diffusion limit. The diffusion coefficient used to calculate $\tau_{D}$ is the geometric mean of $D_{l}$ and $D_{\mu}$ in Figure 8 . The line connecting the calculated values of $\tau_{D}$ at different $L$ is shown dashed between 5.3 and $7.9 R_{J}$ because the radial dependence of $f$ in this region is not known. The curves for $\tau_{s}$ at different values of $M$ were calculated using (5).

$R_{J}$ is consistent with a particle lifetime near the strong pitch angle diffusion limit throughout the inner magnetosphere. This is illustrated in Figure 10, which compares the radial dependence of $\tau_{s}$ with that of the characteristic diffusion time, $\tau_{D}=$ $1 / v_{D}$, where outside $\sim 12 R$, the diffusion time is short in comparison with the particle lifetime so that a low loss rate is expected, whereas inside $\sim 10 R_{J}$ the opposite is true, indicating large losses. The figure also shows that because of the dependence of $\tau_{s}$ on $M$ (equation (5)), one expects ions with smaller magnetic moments to diffuse farther in, on average, before being lost than ions with larger $M$.

In the analysis that follows, we will assume that the predominant particle loss mechanism is pitch angle scattering into the loss cone and therefore that the energetic ions deposit their energy in the Jovian atmosphere. The power delivered to the atmosphere by oxygen ions lost in a given radial range is simply the loss rate of the ions (i.e., the decrease in $F(>M)$ in that radial range from Figure 9) multiplied by their average energy. The average energy $\bar{E}_{M}(L)$ depends on the magnetic moment threshold and is given by

$$
\bar{E}_{M}(L)=\left[\int_{E=M / B}^{\infty} \frac{E}{v} \frac{d J}{d E} d E\right]\left[\int_{E=M / B}^{\infty} \frac{1}{v} \frac{d J}{d E} d E\right]^{-1}
$$

where $v$ is the ion velocity, which is included to transform the flux into a number density.

Figure 11a shows the power per unit $L$ (i.e., per $R_{J}$ ), $d P(>M) / d L$, delivered to the atmosphere by oxygen ions with magnetic moments greater than the indicated thresholds, as a function of the radial distance at which they are lost. Most of the power comes from between 7 and $13 R_{J}$, as expected from Figure 9. Also, there is a shift in the location of the peak power, from $\sim 10.5 R_{J}$ for $>1700 \mathrm{MeV} /$ nuc-G oxygen ions to $\sim 8.5 R_{J}$ for $>70 \mathrm{MeV} /$ nuc-G ions. This is consistent with the shift in the location of the crossover point of $\tau_{s}$ and $\tau_{D}$ in Figure 10. The power per degree latitude has been calculated from the curves in Figure $11 a$ and is shown in Figure $11 b$ as a function of the magnetic latitude at which the power is delivered. The mapping was done using the Voyager 1 magnetic field model of Connerney et al. [1981]. The latitude dependence of the power will be discussed below.

The total power delivered to the atmosphere by oxygen ions with magnetic moments greater than a given $M$ was determined by integrating the curves in Figure $11 a$ and is shown in Figure 12a. The sulfur points in the figure were calculated from the oxygen data using the sulfur to oxygen ratios in Figure 4. The sulfur contribution is approximately equal to that of oxygen
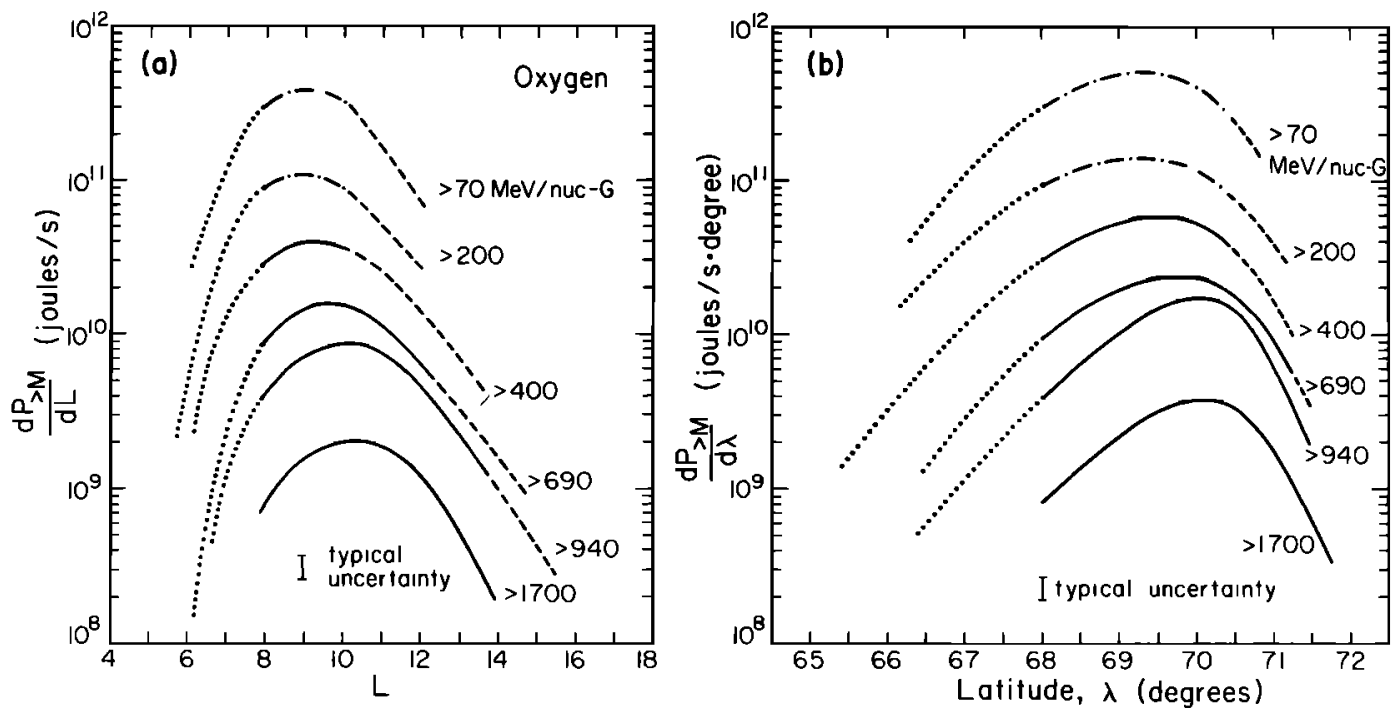

Fig. 11. The power delivered to the Jovian atmosphere (a) per unit $L$ and $(b)$ per degree magnetic latitude by oxygen ions with magnetic moments greater than the indicated values. Typical error bars are shown. The curves in Figure $11 a$ were calculated using (9) and the data in Figure $9 b$. The curves in Figure $11 b$ were calculated from those in Figure $11 a$ using the Voyager 1 magnetic field model of Connerney et al. [1981]. 


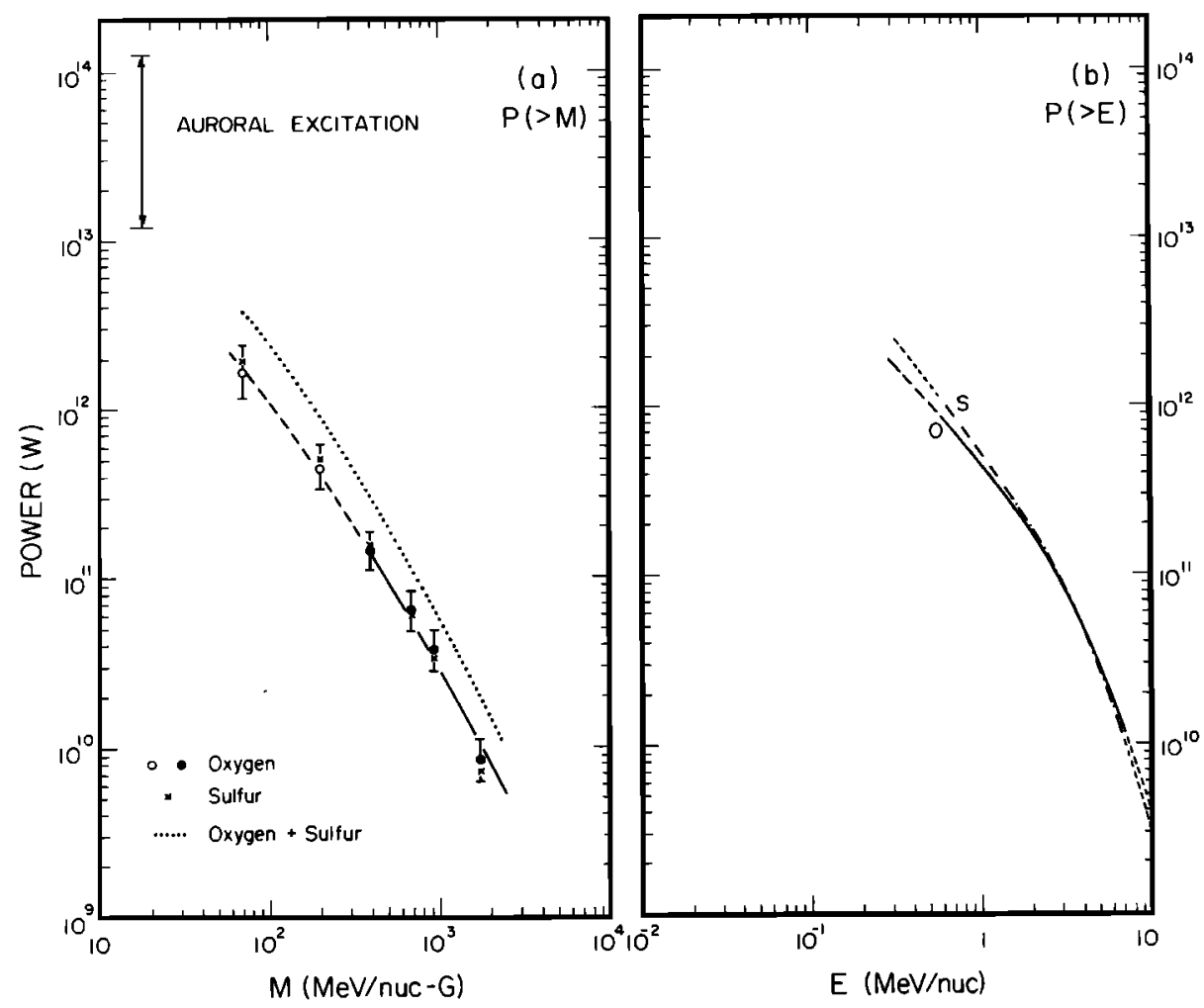

Fig. 12. The total power delivered by oxygen and sulfur ions (a) with magnetic moments greater than $M$ and $(b)$ energy greater than $E$ to the Jovian atmosphere. In Figure $12 a$ the solid circles are based on the measured portions of the spectra in Figure 5 , and the open circles are based on the extrapolated portions. The oxygen points were determined by integrating the curves in Figure 11. The sulfur points were calculated from those of oxygen using the data in Figure 4a. The range of estimates of the power required to excite the observed auroral activity [Broadfoot et al., 1981; Yung et al., 1982] is also shown. In Figure $12 b$ the oxygen curve was calculated from the data in Figure $11 a$. The dashed portions indicate energies at which the calculation is based on extrapolations of the measured data. The sulfur curve was calculated from the oxygen curve using the data in Figure $4 a$.

owing to the fact that the sulfur to oxygen ratio at constant energy per nucleon is $\sim 0.5$ and sulfur has twice as many nucleons. Oxygen and sulfur ions with $>70 \mathrm{MeV} /$ nuc-G are seen to deposit power in excess of $10^{12} \mathrm{~W}$ in the Jovian atmosphere.

The power above a fixed magnetic moment is used in this analysis because it is the quantity best related to the particle diffusion and loss processes. However, for atmospheric studies, a more useful quantity is the power above a fixed energy, $P(>E)$, since a particle's energy determines the depth to which it penetrates into the atmosphere. We have calculated $P(>E)$ as a function of energy from the data in Figure 11a, with the results displayed in Figure $12 b$.

\section{Discussion}

The auroral activity observed on Jupiter is one of the most energetic phenomena associated with the magnetosphere, requiring a continuous power input of $10^{13}-10^{14} \mathrm{~W}$ into the auroral zone [Broadfoot et al., 1981; Yung et al., 1982]. Extrapolation of the curves in Figure 12a suggests that oxygen and sulfur ions with magnetic moments of $\sim 10 \mathrm{MeV} /$ nuc-G ( $\sim 35$ $\mathrm{keV} / \mathrm{nuc}$ at $10 R_{J}$ ) may be delivering a reasonable fraction of that power. From Figure $11 b$, we see that the power from $M$ $\gtrsim 70 \mathrm{MeV} /$ nuc-G is deposited in a $\sim 5^{\circ}$ zone in magnetic latitude between $\sim 67^{\circ}$ and $\sim 72^{\circ}$, which is $\sim 4^{\circ}$ poleward of the Io footprint [Connerney et al., 1981], while ions with lower magnetic moments might precipitate somewhat closer to the footprint. This is consistent with the observed auroral zone width and is in the region in which aurorae are seen [Broadfoot et al., 1981].

From the data in Figure $12 b$, we have estimated the atmospheric depth distribution of the oxygen and sulfur energy deposition, using the tables of Northcliffe and Schilling [1970] to calculate the range of oxygen and sulfur ions in the $\mathbf{H}_{2}$

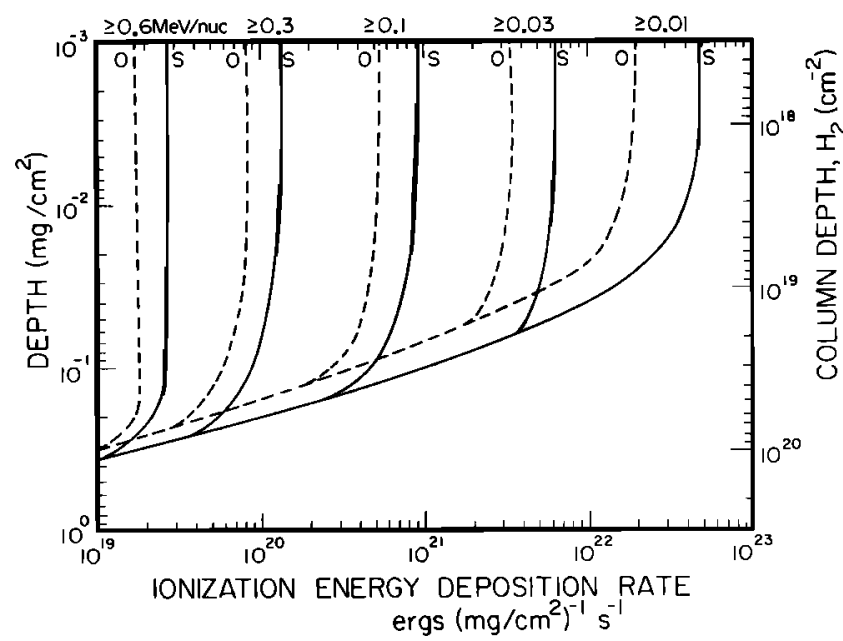

Fig. 13. Illustrative calculation of the energy deposition in the Jovian atmosphere by oxygen and sulfur ions with energies greater than the indicated values, as a function of depth along the particle paths. The curves were calculated by extrapolating the results in Figure $12 b$ to lower energies. 


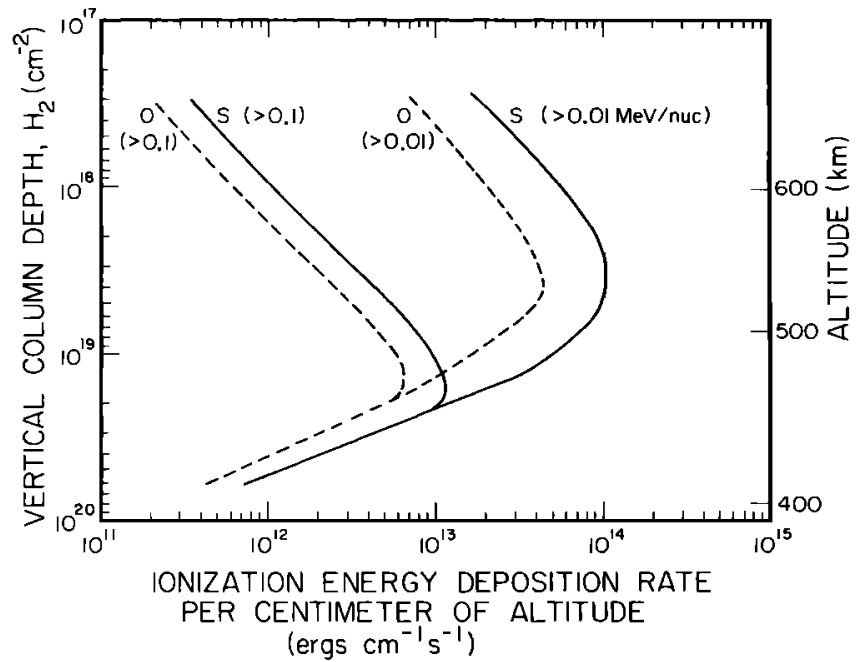

Fig. 14. Illustrative calculation of the altitude dependence of the oxygen and sulfur energy deposition (per centimeter) in the Jovian atmosphere for two lower limits of the extrapolated oxygen and sulfur energy spectra. The curves were calculated from those in Figure 13 using the atmospheric model of Atreya et al. [1981].

region of the upper Jovian atmosphere. The actual spectral forms assumed for the oxygen and sulfur fluences are $F_{0}(>$ $E)=1 \times 10^{23} \quad E^{-2.1}$ oxygen ions $\mathrm{s}^{-1}$ and $F_{s}(>E)=6$ $\times 10^{22} E^{-2.3}$ sulfur ions $\mathrm{s}^{-1}$. For purposes of illustration, it has been assumed that these spectral forms, which are based on observations from $\sim 0.6 \mathrm{MeV} /$ nuc to $4 \mathrm{MeV} /$ nuc, extend to lower energies. Figure 13 shows examples of the oxygen and sulfur energy deposition as a function of depth for cases in which the fluence spectra extend down to lower energy limits ranging from $0.6 \mathrm{MeV} /$ nuc to $0.01 \mathrm{MeV} /$ nuc. The plotted depth is that along the particle path.

As a further illustration, we have calculated the energy deposition as a function of altitude instead of depth, employing the atmospheric model of Atreya et al. [1981] for the number density of molecular hydrogen, as a function of altitude. The effects of the approximately isotropic incident flux on the top of the atmosphere have been taken into account by assuming all particles are incident at $60^{\circ}$. The calculated altitude distribution of deposited energy is shown in Figure 14 for two different energy lower limits to the fluence spectra which were chosen to illustrate the deposition of $\sim 10^{13}$ to $\sim 10^{14} \mathrm{~W}$.

The figure shows that for the assumed spectral extrapolation, most of the energy deposition occurs between $2 \times 10^{18}$ and $5 \times 10^{19} \mathrm{~cm}^{-2}$ of $\mathrm{H}_{2}$, corresponding to an altitude range of approximately $450-550 \mathrm{~km}$. The location of the peak depends on the minimum energy of the spectrum but is above the homopause for either of the two representative lower limits shown in the figure. In their study of the observed wavelength dependence of the ultraviolet auroral emission, Yung et al. [1982] find that the energy deposition must occur between $5 \times 10^{17}$ and $2 \times 10^{20} \mathrm{~cm}^{-2}$, which is consistent with the oxygen and sulfur depth profiles in Figure 14.

The rate at which oxygen ions with $>70 \mathrm{MeV} /$ nuc-G diffuse into the inner magnetosphere is $\sim 10^{24}$ ions/s (Figure 9), which is small in comparison with the $10^{27}-10^{28}$ ions/s created near Io. However, Figure $12 a$ shows that, in order for oxygen and sulfur to drive the aurora, the bulk of the power must come from ions with $\sim 10 \mathrm{MeV} / \mathrm{nuc}-\mathrm{G}$. Since these ions have less energy than those at larger $M$, relatively more are needed for a comparable contribution of power. To estimate the total required particle flow rate, we show in Figure 15 the extrapola- tion to lower magnetic moments of the inward flow rate and power delivered to the atmosphere. The inward flow rate was calculated using the data in Figure 9 (with the sulfur contribution estimated from Figure 4) while the power is from Figure 12. Since the flow rate and power depend on the Io source rate $S_{i}$, the bands indicate the range of values corresponding to $10^{27} \leq S_{i} \leq 10^{28}$ ions/s.

A number of interesting features can be deduced from Figure 15. For example, the observed inward flow rate of oxygen and sulfur ions with $M>70 \mathrm{MeV} /$ nuc-G is $\sim 10^{-3} S_{i}$. Those ions deposit $10^{12}$ to $10^{13} \mathrm{~W}$ in the Jovian atmosphere. Extrapolations to lower magnetic moment suggest, for example, that $\sim 10^{-2} S_{i}$ oxygen and sulfur ions/s may flow inward with $M>30 \mathrm{MeV} / \mathrm{nuc}-\mathrm{G}$. It is also apparent that with the assumed extrapolations, a deposition power of $10^{13}$ to $10^{14} \mathrm{~W}$ could result from an inward flow rate of energetic ions of $\sim 10^{26} \mathrm{~s}^{-1}$. The bulk of these ions would have $M \sim 10$ to $30 \mathrm{MeV} /$ nuc-G, corresponding to $\sim 35$ to $100 \mathrm{keV} /$ nuc at $10 R_{J}$ and $\sim 1.5$ to 5 $\mathrm{keV} / \mathrm{nuc}$ ( $\sim 30$ to $100 \mathrm{keV}$ total kinetic energy) at $40 R_{\text {J }}$.

Ions of these energies are also observed at distances greater than $150 R_{J}$ in the magnetotail, streaming away from Jupiter. The total outward flow rate of this oxygen and sulfur rich magnetosphere wind is estimated to be $\sim 2 \times 10^{27}$ ions/s [Krimigis et al., 1981], indicating that the acceleration process operating in the outer magnetosphere heats a large fraction of the plasma ions that diffuse into the region from Io. It is therefore possible that enough ions are accelerated in the outer magnetosphere to supply the $\sim 10^{26}$ ions/s inward diffusion rate required to power the aurorae.

As a final point, we compare the energy carried into the inner

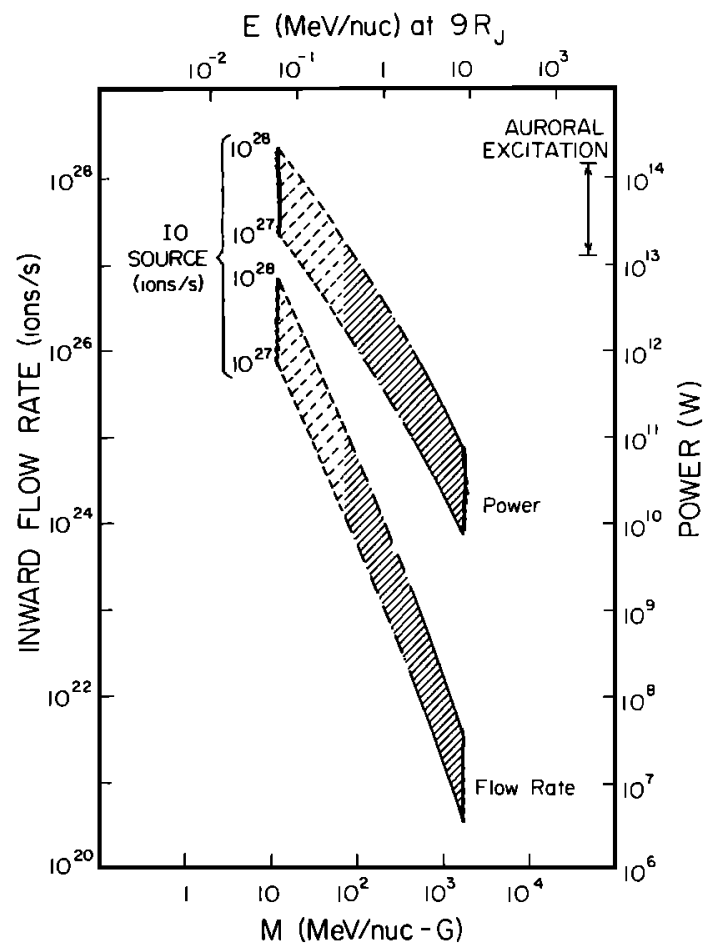

Fig. 15. The inward flow rate of oxygen and sulfur ions across $\sim 15 R_{J}$ (outside the lossy region) and the total power delivered to the atmosphere by these ions, as functions of magnetic moment threshold. The energy of the particles at $9 R_{J}$, which is the typical distance at which the losses occur, is shown on the top axis. The bands indicate the range of values corresponding to an Io source strength of $10^{27}$ to $10^{28}$ ions/s. As in Figure 5, the dot-dash region is derived from LECP spectral shape, while the dashed regions are extrapolations. The auroral excitation power requirement is also indicated. 
magnetosphere by the energetic oxygen and sulfur ions with that flowing outward with the plasma. The inward energy flow rate across $10 R_{J}$ due to $\sim 10^{26}$ ions/s of $\sim 10 \mathrm{MeV} / \mathrm{nuc}-\mathrm{G}$ oxygen and sulfur ions is $\sim 10^{13} \mathrm{~W}$. The number of plasma ions diffusing outward across $10 R_{J}$ is $\sim 5 \times 10^{27}$ ions/s, and their average energy is approximately the corotation energy of an ion with mass 21 amu (the mean atomic mass of the dissociation products of $\mathrm{SO}_{2}$ ), which is $1.7 \mathrm{keV}$. The outward energy flow is therefore $\sim 10^{12} \mathrm{~W}$. Thus if the particle spectrum extends to lower energies as suggested by the extrapolation in Figure 15, then there is a net flow of energy into the inner magnetosphere. The inward energy flow is in fact even larger than $10^{13} \mathrm{~W}$, since the contributions from energetic electrons and protons were not included.

\section{CONCLUSIONS}

The principal conclusions of this study of $Z>2$ ions with energies in the range 1 to $20 \mathrm{MeV} /$ nuc between 5 and $20 R_{J}$ in the Jovian magnetosphere are as follows:

1. The elemental abundances of carbon, sodium, and sulfur relative to oxygen vary as a function of particle magnetic moment but are to first order the same throughout the magnetosphere at a given $M$. For ions with $10^{3} \mathrm{MeV} /$ nuc-G $\left(E \approx 3.5 \mathrm{MeV} /\right.$ nuc at $10 R_{J}$ ) the following abundance ratios are found: $\mathrm{S} / \mathrm{O}=0.5, \mathrm{C} / \mathrm{O}=0.1$, and $\mathrm{Na} / \mathrm{O} \approx 0.05$. The sulfur and sodium energy spectra are softer than the oxygen spectrum, whereas the carbon spectrum is harder, presumably because the carbon is of solar wind origin rather than Iogenic.

2. The diffusion coefficient upper limit at $9 R_{J}$, determined from the phase space density profile of the energetic oxygen ions, is $\sim 10^{-5} \mathrm{~s}^{-1}$. This limit, combined with the analysis of Voyager plasma observations by Siscoe et al. [1981], specifies an upper limit to the mass loading rate near Io of $\sim 10^{28}$ ions/s.

3. Most of the energetic oxygen and sulfur ions are lost as they diffuse inward from 17 to $6 R_{J}$, with the largest losses occurring between 12 and $8 R_{J}$. The particle lifetime throughout the region is within an order of magnitude of the strong pitch angle diffusion lifetime. The location of the maximum losses $\left(8-12 R_{J}\right)$ indicates that neither geometric absorption by Io nor energy loss in the plasma of the Io torus is the dominant loss mechanism. It is postulated that the losses are caused by pitch angle scattering into the loss cone.

4. It is estimated that $10^{24}$ to $10^{25}$ oxygen and sulfur ions with $>70 \mathrm{MeV} /$ nuc-G are lost per second as they diffuse inward from 12 to $8 R_{\mathrm{J}}$. Assuming these ions are scattered into the loss cone, they deliver $10^{12}$ to $10^{13} \mathrm{~W}$ to the Jovian atmosphere. Extrapolations to lower magnetic moments suggest that the $10^{13}-10^{14} \mathrm{~W}$ required to produce the observed auroral emissions [Broadfoot et al., 1981; Yung et al., 1982] may be delivered by oxygen and sulfur ions with magnetic moments of $\sim 10$ to $30 \mathrm{MeV} /$ nuc-G ( $\sim 35$ to $100 \mathrm{keV} /$ nuc at $10 R_{J}$ ) precipitating into the atmosphere at a rate of $\sim 10^{26}$ ions/s. These ions would have a total kinetic energy of $\sim 30$ to $100 \mathrm{keV}$ in the outer magnetosphere, which is approximately the characteristic temperature of the hot plasma in that region [Krimigis et al., 1981].

5. The oxygen and sulfur ions deposit their energy between $\sim 67^{\circ}$ and $\sim 72^{\circ}$ magnetic latitude which is $\sim 4^{\circ}$ poleward of the Io footprint. This is consistent with the observed auroral zone width and is in the region in which aurorae are seen [Broadfoot et al., 1981]. The vertical column depth in the Jovian atmosphere at which the bulk of the power is deposited is $\sim 10^{19} \mathrm{~cm}^{-2}$ of $\mathrm{H}_{2}$, which is within the range of auroral depths derived from the observed wavelength dependence of the ultraviolet emissions [Yung et al., 1982]. This corresponds to a height in the atmosphere of $\sim 500 \mathrm{~km}$, which is above the homopause.

6. If the spectrum extends down to $\sim 10 \mathrm{MeV} /$ nuc-G, then 10 times more energy $\left(\sim 10^{13} \mathrm{~W}\right)$ is carried inward across $10 R_{J}$ by the energetic oxygen and sulfur ions than flows outward with the plasma. Thus the main source of energy for powering the aurora and torus would have to be supplied by an acceleration process occurring in the middle or outer magnetosphere.

Acknowledgments. We greatly appreciate the efforts of R. E. Vogt, both in his capacity as CRS Principal Investigator and as a colleague who has provided useful discussions. We are also indebted to our colleague J. H. Trainor for his contribution to this analysis. We are grateful to the California Institute of Technology and Goddard groups who have supported the investigation, with special thanks to $W$. E. Althouse, R. Burrell, W. R. Cook, and D. E. Stilwell. We also thank D. L. Chenette for helpful discussions. N. F. Ness and his colleagues on the Voyager magnetometer team provided the magnetic field data used in this analysis, which we gratefully acknowledge. We thank Y. L. Yung and G. R. Gladstone for useful discussions concerning the Jovian atmosphere and its auroral emissions. This work was supported in part by NASA under grants NAGW-200 and NGR 05-002-160 and contract NAS7-918 (formerly NAS7-100).

The Editor thanks D. Hamilton and C. K. Goertz for their assistance in evaluating this paper.

\section{REFERENCES}

Atreya, S. K., T. M. Donahue, and M. C. Festou, Jupiter: Structure and composition of the upper atmosphere, Astrophys. J., 247, L43, 1981.

Bagenal, F., and J. D. Sullivan, Direct plasma measurements in the Io torus and inner magnetosphere of Jupiter, J. Geophys. Res., 86, 8447, 1981.

Borovsky, J. E., C. K. Goertz, and G. Joyce, Magnetic pumping of particles in the outer Jovian magnetosphere, J. Geophys. Res., 86 , $3481,1981$.

Broadfoot, A. L., M. J. S. Belton, P. Z. Takacs, B. R. Sandel, D. E. Shemansky, J. B. Holberg, J. M. Ajello, S. K. Atreya, T. M. Donahue, H. W. Moos, J. L. Bertaux, J. E. Blamont, D. F. Strobel, J. C. McConnell, A. Dalgarno, R. Goody, and M. B. McElroy, Extreme ultraviolet observations from Voyager 1 encounter with Jupiter, Science, 204, 979, 1979.

Broadfoot, A. L., B. R. Sandel, D. E. Shemansky, J. C. McConnell, G. R. Smith, J. B. Holberg, S. K. Atreya, T. M. Donahue, D. F. Strobel, and J. L. Bertaux, Overview of the Voyager ultraviolet spectrometry results through Jupiter encounter, J. Geophys. Res., 86, 8259, 1981.

Connerney, J. E. P., M. H. Acuña, and N. F. Ness, Modeling the Jovian current sheet and inner magnetosphere, J. Geophys. Res., 86, 8370 1981.

Eviatar, A., and G. L. Siscoe, Limit on rotational energy available to excite Jovian aurora, Geophys. Res. Lett., 7, 1085, 1980.

Froidevaux, L., Radial diffusion in Io's torus: Some implications from Voyager 1, Geophys. Res. Lett., 7, 33, 1980.

Gehrels, N., E. C. Stone, and J. H. Trainor, Energetic oxygen and sulfur in the Jovian magnetosphere, J. Geophys. Res., 86, 8906, 1981

Goertz, C. K. Energization of charged particles in Jupiter's outer magnetosphere, J. Geophys. Res., 83, 3145, 1978.

Goertz, C. K. Proton aurora on Jupiter's nightside, Geophys. Res. Lett., $7,365,1980$.

Goertz, C. K., J. A. Van Allen, and M. F. Thomsen, Further observational support for the lossy radial diffusion model of the inner Jovian magnetosphere, J. Geophys. Res., 84, 87, 1979.

Hamilton, D. C., G. Gloeckler, S. M. Krimigis, and L. J. Lanzerotti, Composition of nonthermal ions in the Jovian magnetosphere, $J$. Geophys. Res., 86, 8301, 1981.

Kennel, C. F., and H. E. Petschek, Limit on stably trapped particle fluxes, J. Geophys. Res., 71, 1, 1966.

Krimigis, S. M., J. F. Carbary, E. P. Keath, C. O. Bostrom, W. I. Axford, G. Gloeckler, L. J. Lanzerotti, and T. P. Armstrong, Characteristics of hot plasma in the Jovian magnetosphere: Results from the Voyager spacecraft, J. Geophys. Res., 86, 8227, 1981.

Ness, N. F., M. H. Acuña, R. P. Lepping, L. F. Burlaga, K. W. 
Behannon, and F. M. Neubauer, Magnetic field studies at Jupiter by Voyager 1: Preliminary results, Science, 204, 982, 1979a.

Ness, N. F., M. H. Acuña, R. P. Lepping, L. F. Burlaga, K. W. Behannon, and F. M. Neubauer, Magnetic field studies at Jupiter by Voyager 2: Preliminary results, Science, 206, 966, 1979 b.

Northcliffe, L. C., and R. F. Schilling, Range and stopping-power tables for heavy ions, Nucl. Data Tables, A7, 233, 1970.

Schulz, M., and L. J. Lanzerotti, Particle Diffusion in the Radiation Belts, Springer, New York, 1974.

Shemansky, D. E., Radiative cooling efficiencies and predicted spectra of species of the Io plasma torus, Astrophys. $J ., 236,1043,1980 a$.

Shemansky, D. E., Mass-loading and the diffusion-loss rates of the Io plasma torus, Astrophys. J., 242, 1266, $1980 b$.

Shemansky, D. E., and B. R. Sandel, The injection energy into the Io plasma torus, J. Geophys. Res., 87, 219, 1982.

Siscoe, G. L., A. Eviatar, R. M. Thorne, J. D. Richardson, F. Bagenal, and J. D. Sullivan, Ring current impoundment of the Io plasma torus, J. Geophys. Res., 86, 8480, 1981.

Stilwell, D. E., W. D. Davis, R. M. Joyce, F. B. McDonald, J. H. Trainor, W. E. Althouse, A. C. Cummings, T. L. Garrard, E. C. Stone, and R. E. Vogt, The Voyager cosmic ray experiment, IEEE Trans. Nucl. Sci., NS-26, 513, 1979.

Stone, E. C., R. E. Vogt, F. B. McDonald, B. J. Teegarden, J. H. Trainor, J. R. Jokipii, and W. R. Webber, Cosmic ray investigation for the Voyager missions: Energetic particle studies in the outer heliosphere-and beyond, Space Sci. Rev., 21, 355, 1977.

Sullivan, J. D., and G. L. Siscoe, In situ observations of lo torus plasma, in The Satellites of Jupiter, edited by D. Morrison, University of Arizona Press, Tucson, 1981.
Thomsen, M. F., C. K. Goertz, and J. A. Van Allen, A determination of the $L$ dependence of the radial diffusion coefficient for protons in Jupiter's inner magnetosphere, J. Geophys. Res., 82, 3655, 1977.

Thorne, R. M., Jovian auroral secondary electrons and their influence on the Io plasma torus, Geophys. Res. Lett., 8, 509, 1981 .

Thorne, R. M., Microscopic plasma processes in the Jovian magnetosphere, in Physics of the Jovian Magnetosphere, edited by A. J. Dessler, Cambridge University Press, New York, 1981b.

Thorne, R. M., Injection and loss mechanisms for energetic ions in the inner Jovian magnetosphere, J. Geophys. Res., 87, 8105, 1982.

Thorne, R. M., and B. T. Tsurutani, Diffuse Jovian aurora influenced by plasma injection from Io, Geophys. Res. Lett., 6, 649, 1979.

Vogt, R. E., W. R. Cook, A. C. Cummings, T. L. Garrard, N. Gehrels, E. C. Stone, J. H. Trainor, A. W. Schardt, T. Conlon, N. Lal, and F. B. McDonald, Voyager 1: Energetic ions and electrons in the Jovian magnetosphere, Science, 204, 1003, 1979a.

Vogt, R. E., A. C. Cummings, T. L. Garrard, N. Gehrels, E. C. Stone, J. H. Trainor, A. W. Schardt, T. F. Conlon, and F. B. McDonald, Voyager 2: Energetic ions and electrons in the Jovian magnetosphere, Science, 206, 984, $1979 b$.

Yung, Y. L., G. R. Gladstone, K. M. Chang, J. M. Ajello, and S. K. Srivastava, $\mathrm{H}_{2}$ fluorescence spectrum from 1200 to $1700 \AA$ by electron impact: Laboratory study and application to Jovian aurora, Astrophys. J., 254, L65, 1982.

(Received November 30, 1982; revised March 25, 1983; accepted April 11, 1983.) 